\title{
General design equations for the rotational stiffness, maximal angular deflection and rotational precision of various notch flexure hinges
}

\author{
Sebastian Linß ${ }^{1}$, Philipp Schorr ${ }^{2}$, and Lena Zentner ${ }^{1}$ \\ ${ }^{1}$ Mechanism Technology Group, Technische Universität Ilmenau, Ilmenau, 98693, Germany \\ ${ }^{2}$ Technical Mechanics Group, Technische Universität Ilmenau, Ilmenau, 98693, Germany \\ Correspondence to: Sebastian Linß (sebastian.linss@tu-ilmenau.de)
}

Received: 15 December 2016 - Revised: 17 January 2017 - Accepted: 25 February 2017 - Published: 15 March 2017

\begin{abstract}
Notch flexure hinges are often used as revolute joints in high-precise compliant mechanisms, but their contour-dependent deformation and motion behaviour is currently difficult to predict. This paper presents general design equations for the calculation of the rotational stiffness, maximal angular elastic deflection and rotational precision of various notch flexure hinges in dependence of the geometric hinge parameters. The novel equations are obtained on the basis of a non-linear analytical model for a moment and a transverse force loaded beam with a variable contour height. Four flexure hinge contours are investigated, the semi-circular, the cornerfilleted, the elliptical, and the recently introduced bi-quadratic polynomial contour. Depending on the contour, the error of the calculated results is in the range of less than $2 \%$ to less than $16 \%$ for the suggested parameter range compared with the analytical solution. Finite elements method (FEM) and experimental results correlate well with the predictions based on the comparatively simple and concise design equations.
\end{abstract}

\section{Introduction}

In precision engineering applications, micromechanical systems or special applications often compliant mechanisms (Howell, 2013; Zentner, 2014) are used instead of rigidbody mechanisms. Due to their monolithic design, compliant mechanisms are suitable to realize high reproducible motion without clearance, external friction and wear. In compliant mechanisms with lumped compliance mostly flexure hinges (Lobontiu, 2003) are used as material coherent revolute joints. Among a variety of flexure hinge types the notch flexure hinge is state of the art. Therefore, lots of cut-out geometries are described in literatures, while the circular or corner-filleted flexure hinge contour are mainly used in technical systems.

Based on the rigid-body model (Howell and Midha, 1994) the rigid-body replacement method is widely used for the synthesis of compliant mechanisms, especially in precision engineering. In comparison to the optimal synthesis with a continuum model, a better guiding accuracy of a coupler point is possible with this method (Pavlović et al., 2010).
In contrast to the synthesis of rigid-body mechanisms the stress and deformation behaviour as well as the motion behaviour must be considered as multi-objective design criteria in the compliant mechanisms synthesis. Starting from the rigid-body mechanism, this leads to a complex and iterative model-based design process for compliant mechanisms. Therefore, usually numerical methods and simulations are needed. Regarding the required mechanism properties, the step of the geometric design of the notch flexure hinges is a key aspect in the synthesis of a compliant mechanism. Therefore, many different approaches exist, but nevertheless no detailed guidelines or concise design equations for the contourdependent and multi-criterial calculation of the flexure hinge characteristics are known to the author. Existing design equations are not concise, have complex structural form, and are limited to commonly used hinge contours (cf. Sect. 4). In addition, the rotational precision depends on the approach to model the position of the rotation axis during motion. Hence, a suitable approach for the model-based investigations must be used. In conclusion, simple and concise design equations for flexure hinges would be of great benefit to the accelerated 
and goal-oriented synthesis of compliant mechanisms without computer-aided simulations or the test of manufactured prototypes.

Regarding a plane rotational motion due to a bending moment or a transverse force load, this paper addresses the development of new general design equations for the simplified calculation of the rotational stiffness, maximal angular elastic deflection and rotational precision of various notch flexure hinges with a semi-circular, corner-filleted, elliptical, and polynomial contour for an appropriate range of the basic hinge parameters. The design equations are derived according to results of the analytical solution based on the nonlinear theory for modelling of large deflections of rods.

The remaining sections are organized as follows. In Sect. 2, the state of the art of flexure hinges and their geometrical notch design is presented together with the investigated flexure hinge contours in this paper. In Sect. 3, the analytical characterization of the flexure hinges is described regarding the three mentioned performance criteria in dependence of the hinge contour and the geometric parameters. In Sect. 4, the method and results for the derived design equations are presented. In Sect. 5, the equation-based results are discussed and compared with the exact analytical solution and the results of a FEM-based and experimental characterization of the regarded flexure hinges. Finally, conclusions are drawn in Sect. 6.

\section{Design of the flexure hinges}

In contrast to form- and force-closed joints a flexure hinge enables a restoring force which can be advantageous in technical systems (this performance criterion is named rotational stiffness). According to the material coherent connection, the angular deflection of a flexure hinge is limited by reaching admissible material stress respective elastic strain values (maximal angular deflection). Thus, the motion range of a compliant mechanism is limited too? by the hinge in the kinematic chain with largest rotation angle. In addition, no exact relative rotation is possible with a flexure hinge because always a shift of its axis of rotation occurs in dependence of geometric and load parameters (rotational precision). In turn, this can lead to path deviations of the compliant mechanism compared to the rigid-body mechanism, which are not negligible especially in precision engineering (Venanzi et al., 2005; Linß et al., 2014).

As a flexure hinge in this paper a monolithic, small-length and elastic deformable segment of a compliant mechanism is meant, which realizes the function of a relative rotation of two adjacent links mainly due to bending. The demand for a larger angular deflection and a low shift of the rotational axis during the rotation results in a variety of sometimes very complex flexure hinge types, like the butterfly hinge (e.g. Henein et al., 2003; Pei and Xu, 2011) for example. However, the investigations in this paper are focused on notch flexure hinges. Due to their low complexity they are easy to manufacture and therefore mainly used in plane compliant mechanisms, especially for kinematic chains with a higher link number. Furthermore, notch flexure hinges enable optimization potential regarding the rotational precision and possible deflection as equivalent objectives, which is not used yet. Hence, generalized design equations would be of a great benefit.

\subsection{Existing approaches for designing the notch geometry of a flexure hinge}

In the past, notch flexure hinges have been designed very frequently so that various cut-out geometries are proposed to describe the hinge contour, see Fig. 1. Mostly, there are predefined basic geometry elements, which lead to three main notch flexure hinge types, each with a typical characteristic: The precise hinge with a semi-circular contour (e.g. Paros and Weisbord, 1965; Wu and Zhou, 2002), the largedeflective hinge with a corner-filleted contour (e.g. Lobontiu, 2003; Meng et al., 2013) or the elliptical hinge (e.g. Smith et al., 1997; Chen et al., 2008) as a compromise. Furthermore, flexure hinges are designed with other elementary geometries to realize a special characteristic, like the parabolic or hyperbolic contour (e.g. Lobontiu, 2003; Chen et al., 2009), and cycloidal contour (Tian et al., 2010). Increasingly flexure hinges are designed with a combination of the mentioned basic geometries (e.g. Zelenika et al., 2009; Lobontiu et al., 2011; Chen et al., 2011). Rarely special mathematical functions are used that allow more precise shape variations of the partial or whole hinge contour due to a higher number of geometric parameters, like the spline contour (Christen and Pfefferkorn, 1998; De Bona and Munteanu, 2005), the powerfunction contour (Li et al., 2013), the exponent-sine contour (Wang et al., 2013), the Lamé contour (Desrochers, 2008), and the Bézier contour (Vallance et al., 2008). The design with undefined freeform geometries based on topology optimization (Zhu et al., 2014) is a very complex, non-intuitive and not a general design process.

Nevertheless, special higher order polynomial functions, which was suggested by author (Linß et al., 2011b), are not state of the art. Among the variety of cut-out geometries especially polynomial contours offer high potential for optimization while a comparatively simple contour modelling is possible. Depending on the polynomial order and the coefficients arbitrary complex curves can be realized. Furthermore, nearly any elementary geometry could be approximated.

While usually completely symmetric flexure hinges are used, there are a several studies on transversal and axial symmetric hinges. Especially axial symmetric flexure hinges are realised mostly as so-called hybrid hinges because they allow combining the advantages of right circular and corner-filleted flexure hinges (Chen et al., 2005). Further it is known, that a better kinematic behaviour up to an ideal rotation axis can be realized due to a smaller radius at the loaded hinge side 


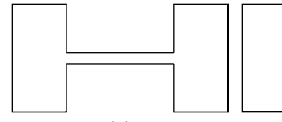

(a)

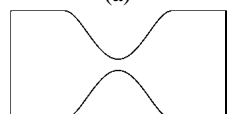

(g)

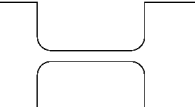

(b)

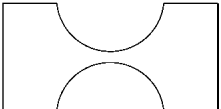

(c)

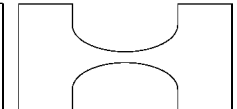

(d)

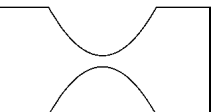

(e)

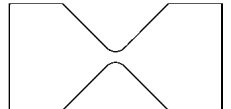

(f)

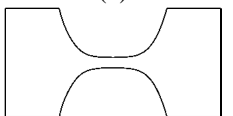

(h)

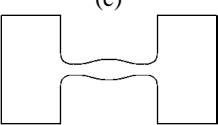

(i)

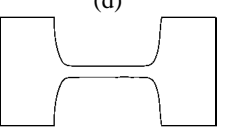

(j)

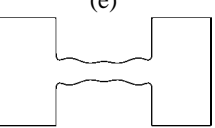

(k)

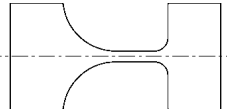

(l)

Figure 1. Approaches for the geometric design of a flexure hinge contour with notches on both sides: (a) rectangular, (b) corner-filleted, (c) semi-circular, (d) elliptical, (e) parabolic, (f) hyperbolic, (g) cycloidal, (h) polynomial higher order, (i) spline function, (j) special mathematic functions, (k) freeform, (l) axial symmetric with transversal asymmetry.

(Linß et al., 2011a; Lin et al., 2013). However, in this paper only transversal and axial symmetric notch flexure hinges are investigated at first because they allow a holistic and intuitive design with regard to the mechanism synthesis.

\subsection{Regarded flexure hinge and investigated hinge contours}

Subject of the investigations in this paper is a separate notch flexure hinge, which is fixed at one end, see Fig. 2a. A given moment $\boldsymbol{M}=-\boldsymbol{M} \boldsymbol{e}_{z}$ or transverse force $\boldsymbol{F}=-F \boldsymbol{e}_{y}$ leads to an angular deflection of the free end with the rotation angle $\varphi$.

The variable hinge contour height $h_{\mathrm{n}}(x)$ is defined by the chosen notch geometry. As it is known, that a deformation not only occurs in the notch segment (Zettl et al., 2005; Yong et al., 2008), the flexure hinge is always modelled with little segments of the both adjacent links. Regarding the influence on the flexure hinge properties, two groups of geometric design parameters are investigated: The basic hinge dimensions $(l, L, h, H, b)$ and the hinge contour (function $h_{\mathrm{n}}(x)$ ).

The determination of typical functional parameters of the hinge contour, e.g. radii, can be done by selection, design of experiments, or optimization, where this design step is not in the focus of this paper. For the investigations, a flexure hinge with the following characteristics of the hinge contour $h_{\mathrm{n}}(x)$ is regarded: A symmetric, continuously differentiable, not undercut contour with the minimal notch height $h$ in the middle (at $x=0$ ), and with a rectangular cross-section. The total height $H$ (which represents the link height in a compliant mechanism too) as well as the total length $L=2 \mathrm{H}$ are chosen to be constant for all investigations. Thus, the distance of the acting load to the middle of the hinge always is the half-length of $L$ to ensure comparability. The hinge length $l$, the minimal hinge height $h$, and the hinge width $b$ are varied within the design domain according to the introduced dimensionless ratios $\beta_{l}, \beta_{h}$, and $\beta_{b}$ with

$\beta_{l}=\frac{l}{H}, \beta_{h}=\frac{h}{H}$, and $\beta_{b}=\frac{b}{H}$.

The exact notch length $l_{\mathrm{n}}$ depends on the notch contour and is mostly equal to the hinge length $l$, as described in the following subsections. For the investigations in this paper four flexure hinge contours are considered (see Fig. 2b), the three typical corner-filleted, semi-circular and elliptical contours, and the bi-quadratic polynomial contour of 4 th order.

\subsubsection{Corner-filleted contour}

There exist different suggestions for the suitable design of the geometric parameters of corner-filleted flexure hinges aiming for low stress values by using a special ratio of the fillet radius and the minimal hinge height, e.g. $r=0.64 h$ (Wittwer et al., 2004), $r=0.7 h$ (Schotborgh et al., 2005), or $r>2 h$ (Henein, 2011). As previous results show, also a stress optimal fillet radius relating to the hinge length can be generalized with $r=0.1 l$ ( $\operatorname{Lin} \beta$ et al., 2011a). This ratio is used by Schotborgh et al. (2005) and Meng et al. (2013) too.

Therefore corner-filleted contours with a stress optimal fillet radius of $r=0.1 l$ are always regarded in this paper. Hence, for the investigated flexure hinges there is a continuously intersection of the circular arc with the both adjacent horizontal and vertical lines, see Fig. 3. The used contour function $h_{\mathrm{n}}(x)$ for all corner-filleted flexure hinges is sectional expressed by Eq. (2), and the notch length $l_{\mathrm{n}}$ always equals the hinge length $l$.

$h_{\mathrm{n}}(x)=\left\{\begin{array}{l}h+2 r-2 \sqrt{r^{2}-\left(x+\frac{l}{2}-r\right)^{2}},-\frac{l}{2} \leq x<-\frac{l}{2}+r \\ h,-\frac{l}{2}+r \leq x \leq \frac{l}{2}-r \\ h+2 r-2 \sqrt{r^{2}-\left(x-\frac{l}{2}+r\right)^{2}}, \frac{l}{2}-r<x \leq \frac{l}{2}\end{array}\right.$

\subsubsection{Semi-circular contour}

To ensure principle similarity of circular contours for the varying hinge dimensions $l$ and $h$, in this work flexure hinges with the radius $R=0.5 l$ are always considered. In dependence of $h$, this leads to contours which consist of an exact (cf. Fig. 4a and b) or approximated (cf. Fig. 4c) semi-circular arc. Therefore, three different models of a semi-circular flexure hinge have become necessary.

According to this, the notch length $l_{\mathrm{n}}$ can be calculated with Eq. (3). The used contour function $h_{\mathrm{n}}(x)$ for flexure 


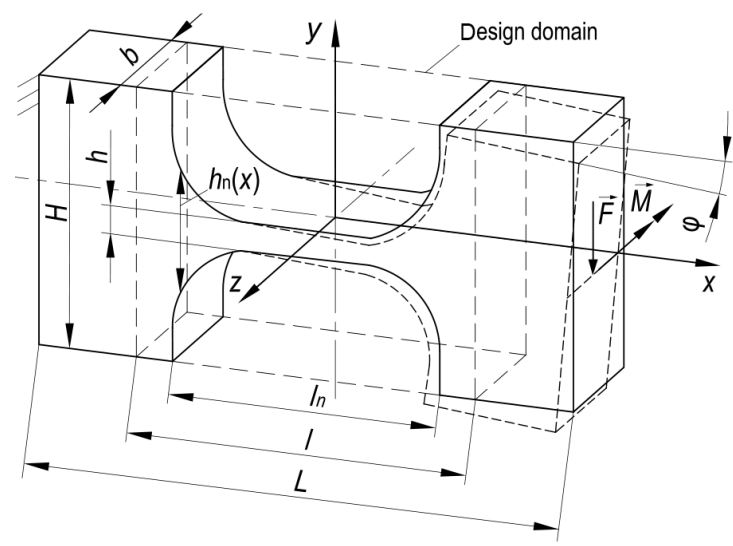

(a)

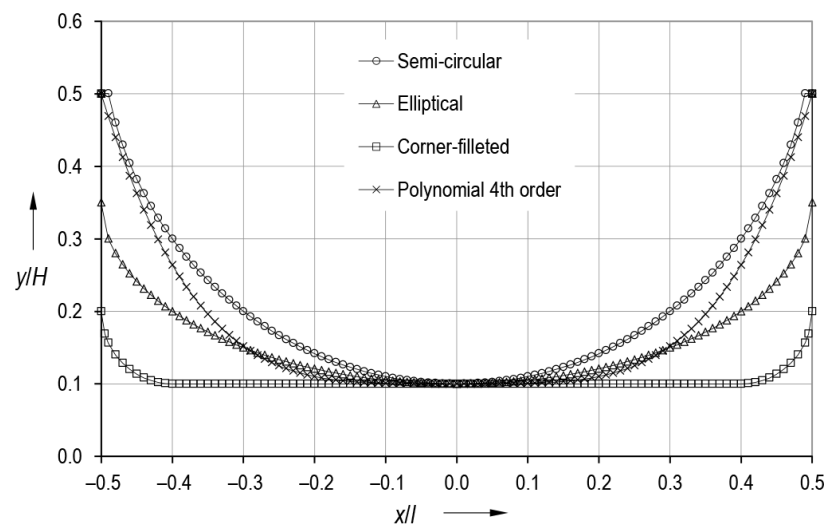

(b)

Figure 2. Investigated flexure hinge: (a) geometric parameters and deflected state as a result of a moment or a force load, (b) representation of $h_{\mathrm{n}}(x)$ for four different flexure hinge contours (half joint, $\beta_{l}=1, \beta_{h}=0.2$ ).

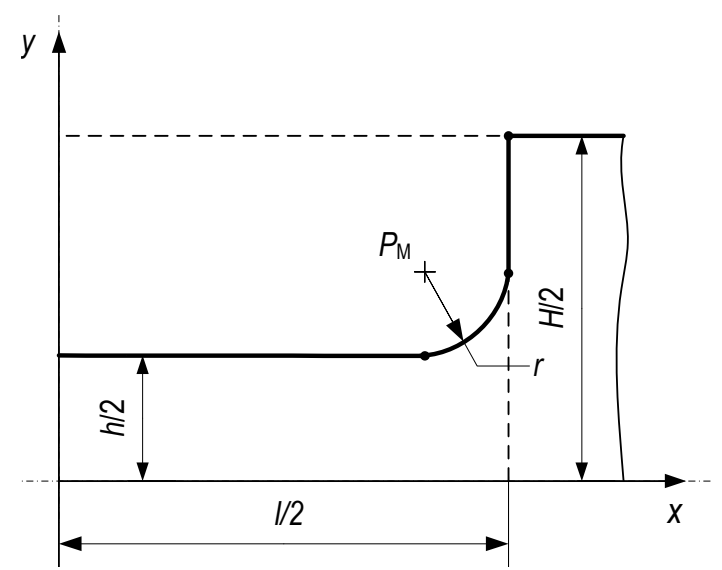

Figure 3. Quarter model of a flexure hinge with a corner-filleted contour with the stress optimal fillet radius $r=0.1 l$.

hinges with exact semi-circular $\operatorname{arcs}(2 R \leq H-h)$ is expressed by Eq. (4). Otherwise the contour is expressed in sections.

$l_{\mathrm{n}}=\left\{\begin{array}{l}l, R \leq \frac{H-h}{2} \\ 2 \sqrt{R^{2}-\left(\frac{H-h}{2}-R\right)^{2}}, R>\frac{H-h}{2}\end{array}\right.$

$h_{\mathrm{n}}(x)=h+2 R-2 \sqrt{R^{2}-x^{2}}$

\subsubsection{Elliptical contour}

For modelling similar elliptical contours the same approach is used as for the semi-circular contours. Next, the elliptical flexure hinges are always considered with the two radii $r_{x}=$ $0.5 l$ and $r_{y}=0.25 l$. Hence, there are three different models of an elliptical hinge in dependence of $h$, see Fig. 5.
According to this, the notch length $l_{\mathrm{n}}$ can be calculated with Eq. (5). The used contour $h_{\mathrm{n}}(x)$ for flexure hinges with exact semi-elliptical arcs $\left(r_{x} \leq H-h\right)$ is expressed by Eq. (6). Otherwise the contour is expressed in sections.

$l_{\mathrm{n}}=\left\{\begin{array}{l}l, r_{x} \leq H-h \\ 2 r_{x} \sqrt{1-\frac{\left(\frac{H-h}{2}-r_{y}\right)^{2}}{r_{y}^{2}}}, r_{x}>H-h\end{array}\right.$

$h_{\mathrm{n}}(x)=h+2 r_{y}\left(1-\sqrt{1-\frac{x^{2}}{r_{x}^{2}}}\right)$

\subsubsection{Polynomial contour}

For modelling flexure hinges special polynomial contours are suitable too ( $\operatorname{Lin} \beta$ et al., 2011b). In this case, the contour function $h_{\mathrm{n}}(x)$ with an even polynomial order $n$ is expressed by Eq. (7). An odd polynomial order can be also achieved if the equation is only used for a quarter hinge, which has to be mirrored twice in the CAD model afterwards.

$h_{\mathrm{n}}(x)=h+\frac{(H-h)}{\left(\frac{l}{2}\right)^{n}} x^{n}$

Based on FEM simulations the influence of the polynomial order $n$ (see Fig. 6) on the hinge properties has been investigated (Linß, 2015). For a given deflection angle it has been shown, that 16th-order polynomial contours lead to comparable low stress values like corner-filleted contours. Furthermore it was found, that 4th-order polynomial contours are suitable to realize both, a precise rotation with low stress in general. Therefore these bi-quadratic polynomial contours are used for the following investigations and the development of design equations. In addition, a further direct optimization 


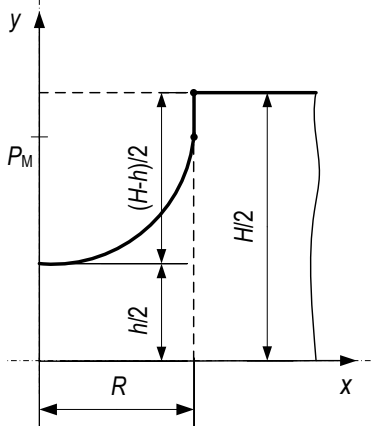

(a)

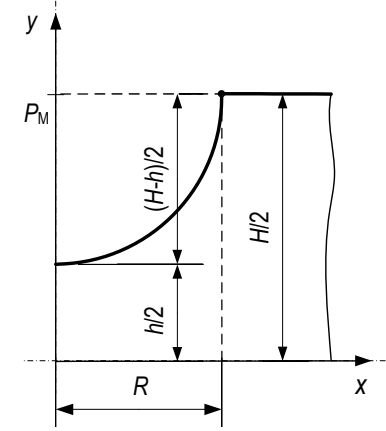

(b)

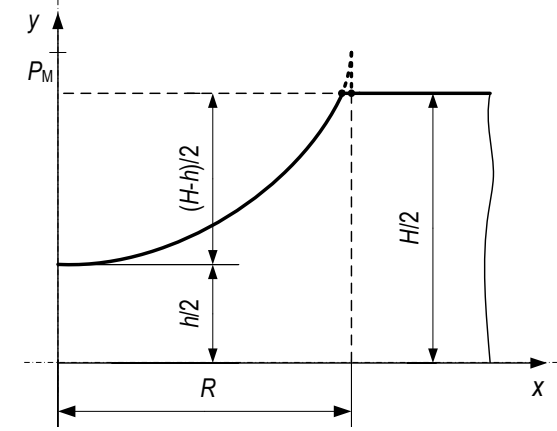

(c)

Figure 4. Quarter model of a flexure hinge with a semi-circular contour with $R=0.5 l$ : (a) if $2 R<H-h$, (b) if $2 R=H-h$ (right semicircular contour), (c) if $2 R>H-h$.

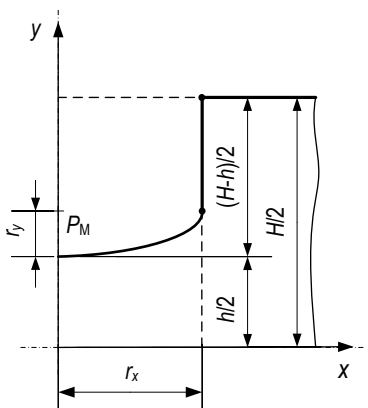

(a)

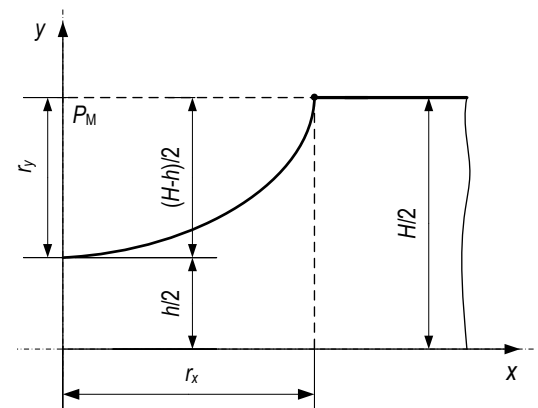

(b)

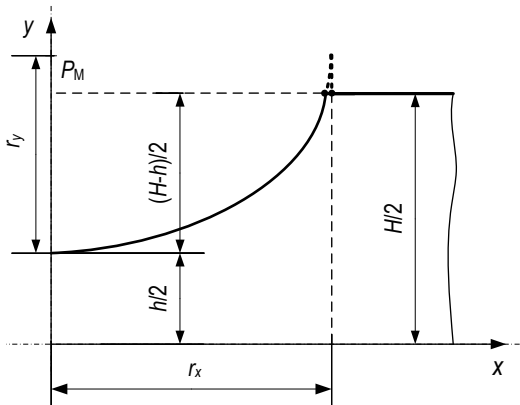

(c)

Figure 5. Quarter model of a flexure hinge with an elliptical contour with $r_{x}=0.5 l$ and $r_{y}=0.25 l$ : (a) if $r_{x}<H-h$, (b) if $r_{x}=H-h$ (right elliptical contour), (c) if $r_{x}>H-h$.

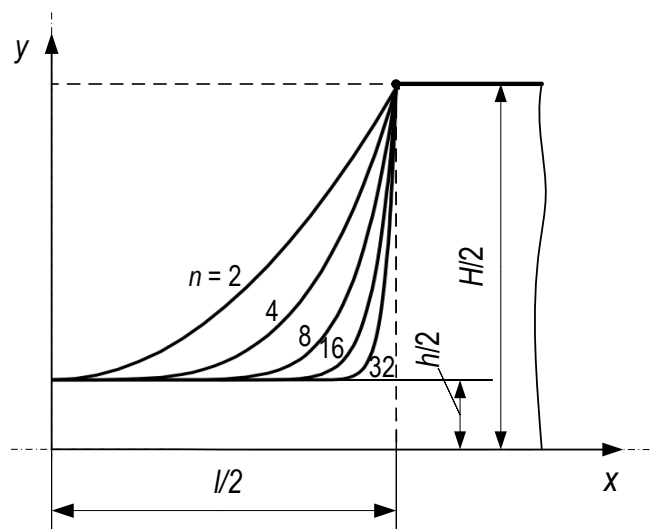

Figure 6. Quarter model of a flexure hinge with polynomial contours of a different order $n$.

of the order $n$ in compliant mechanisms with different hinges in one mechanism is possible (Linß et al., 2015).

The used 4th-order polynomial contour function $h_{\mathrm{n}}(x)$ is expressed by Eq. (8).

$h_{\mathrm{n}}(x)=h+\frac{(H-h)}{\left(\frac{l}{2}\right)^{4}} x^{4}$

\section{Analytical characterization of the flexure hinges}

In this section, the approach of the non-linear analytical characterization of a notch flexure hinge and the results for its rotational stiffness, strain distribution, maximal angular elastic deflection, and rotational precision are presented in dependence of the hinge contour and the geometric parameters $\beta_{l}$, $\beta_{h}$, and $\beta_{b}$.

The analytical characterization is based on the non-linear theory for modelling large deflections of curved rods, e.g. Zentner (2014), for which the dimensions of a cross-section are small compared to the rod length. Equilibrium equations are used to describe a rod element on the basis of the assumptions of a static problem for a slender structure with an axial inextensible line and the val idity of Bernoulli hypothesis, Saint-Venant's principle, and Hooke's law. Thus, for describing a flexure hinge as a beam four non-linear differential 


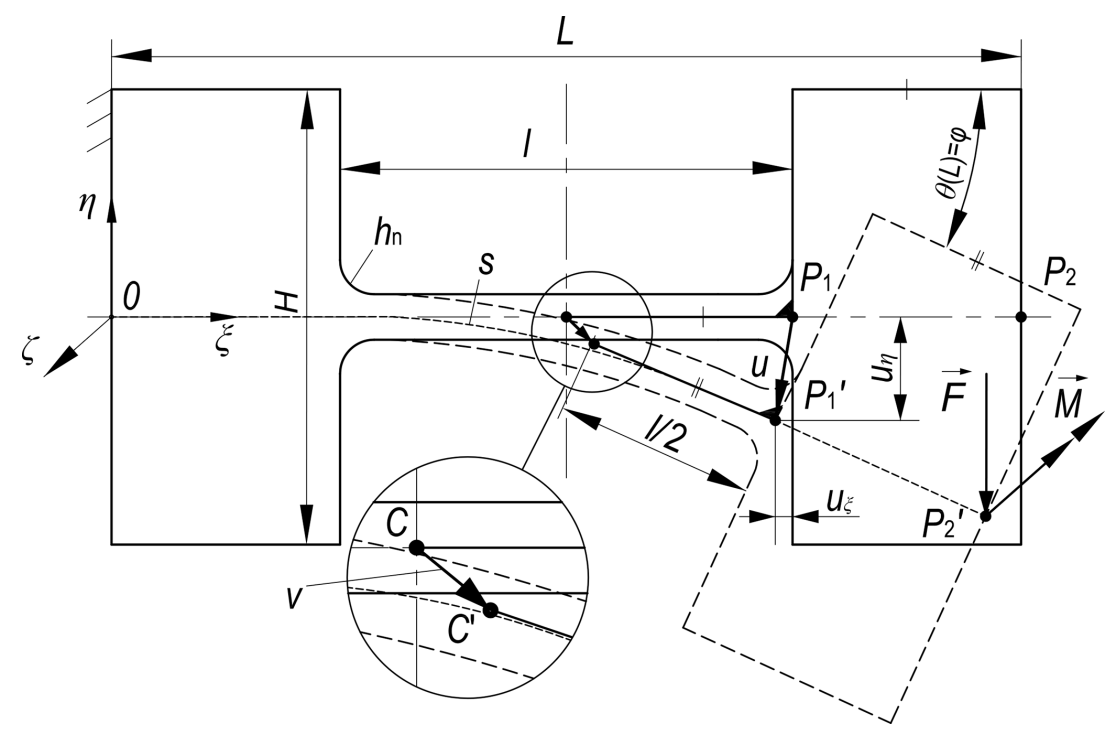

Figure 7. Parameters of the characterized flexure hinge (drawing of the initial and deflected position) with the model for the determination of the rotational axis shift based on guiding the centre with a constant distance (fixed centre approach).

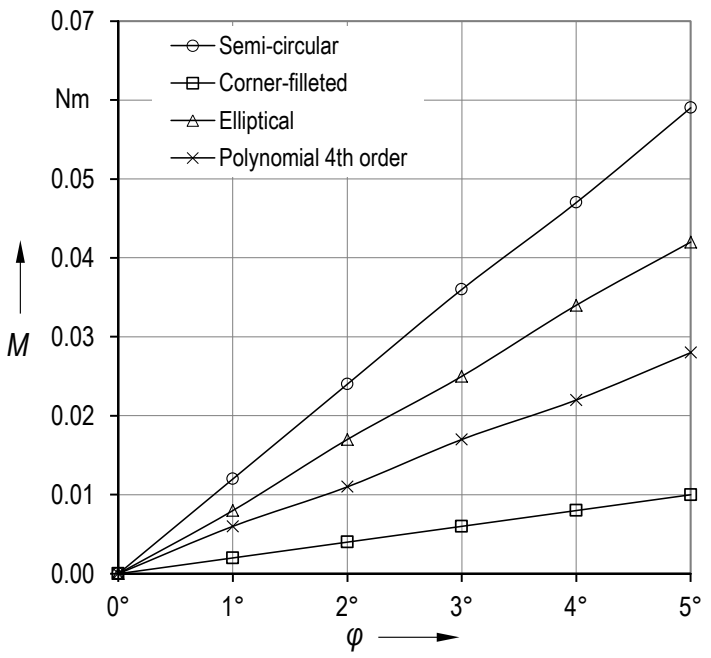

(a)

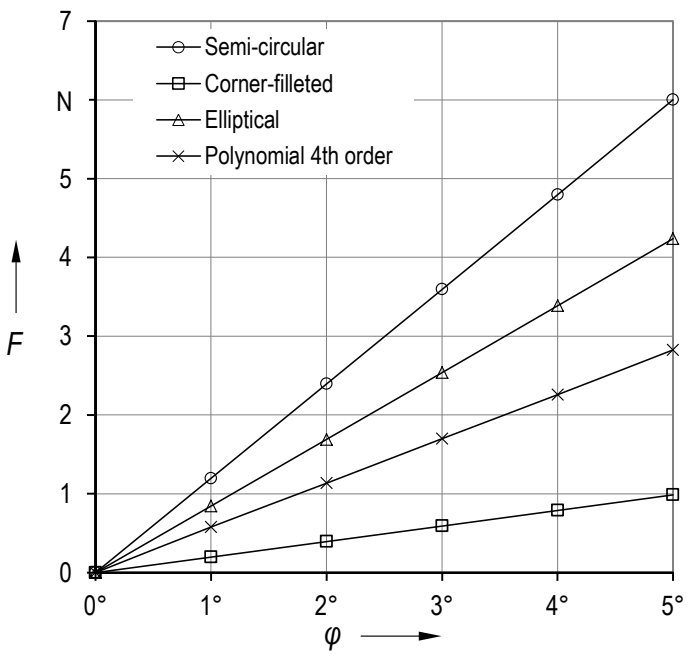

(b)

Figure 8. Influence of the hinge contour - analytical results for the rotational stiffness of a flexure hinge with various contours $\left(\beta_{l}=1\right.$, $\beta_{h}=0.03, \beta_{b}=0.6$ ): (a) moment load, (b) force load.

equations result:

$\frac{\mathrm{d} M}{\mathrm{~d} s}+F \cos \theta=0$,

$\frac{\mathrm{d} \theta}{\mathrm{d} s}-\kappa=0$, with $\kappa=\frac{M}{E I_{\zeta}}$ and $I_{\zeta}=\frac{b h_{\mathrm{n}}^{3}}{12}$,

$\frac{\mathrm{d} u \xi}{\mathrm{d} s}-\cos \theta+1=0$

$\frac{\mathrm{d} u_{\eta}}{\mathrm{d} s}-\sin \theta=0$.

The model considers a stationary coordinate system $\xi \eta \zeta$ with the origin $O$ at the neutral axis at the fixed end of the flexure hinge, see Fig. 7. The parameter $s$ describes the arc length of the neutral axis at the regarded cross-section point and it represents the coordinate of the axial beam line, which is equal to the $\xi$ axis only for the non-deflected hinge. Since the flexure hinge is symmetric, no initial curvature of the beam is regarded in this paper. Hence, at every point $s$ of the beam, the curvature $\kappa$ describes the gradient of the bending angle $\theta$ as a result of the given moment or force load. Due to the non-linear differential equation system, closed-form equations which describe the deflected state cannot be derived. However, for the regarded hinge, which is fixed at one 


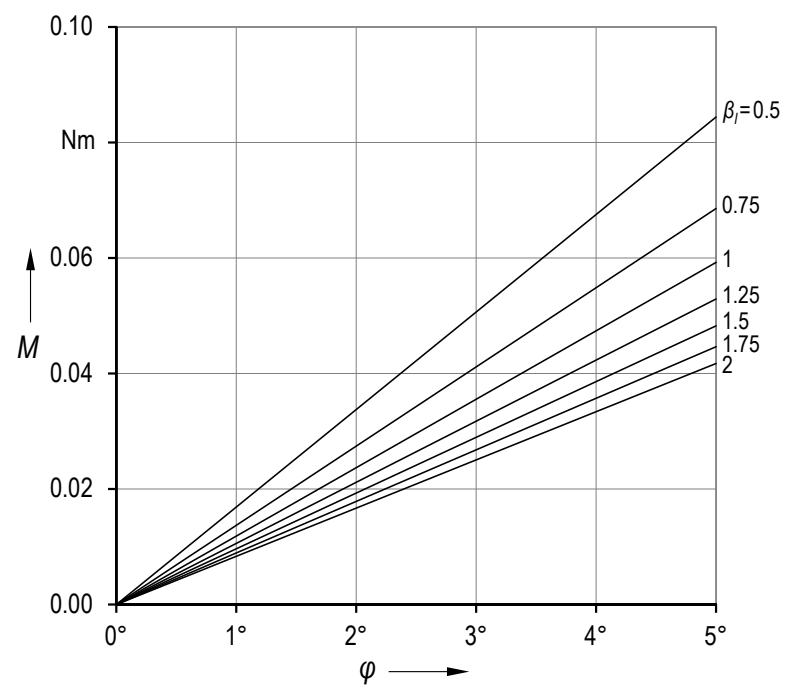

(a)

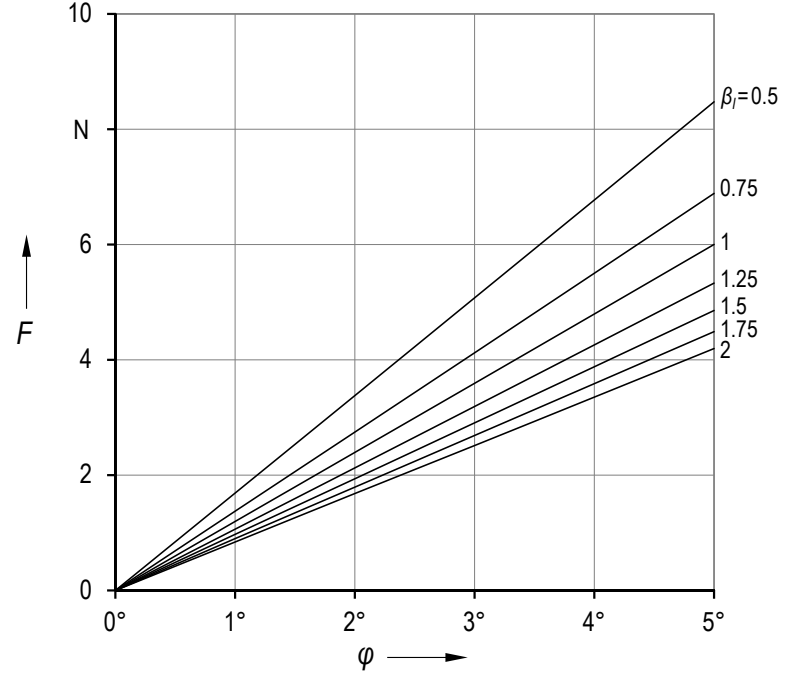

(b)

Figure 9. Influence of the hinge dimensions - analytical results for the influence of $\beta_{l}$ on the rotational stiffness of a semi-circular flexure hinge $\left(\beta_{h}=0.03, \beta_{b}=0.6\right)$ : (a) moment load, (b) force load.

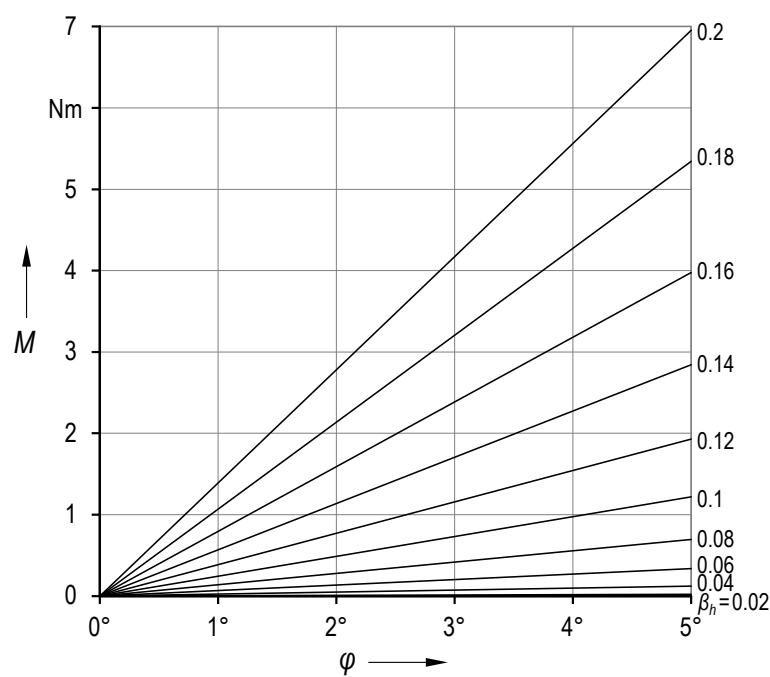

(a)

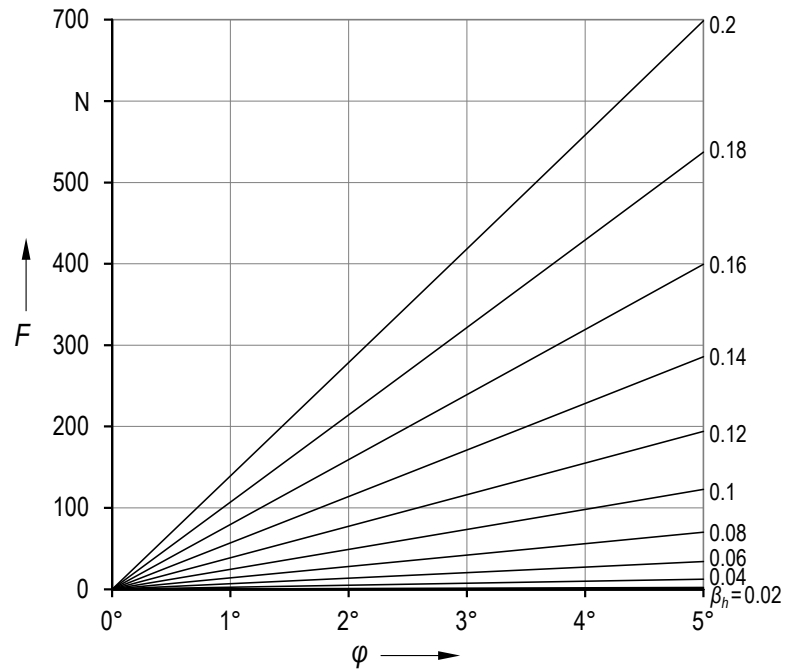

(b)

Figure 10. Influence of the hinge dimensions - analytical results for the influence of $\beta_{h}$ on the rotational stiffness of a semi-circular flexure hinge $\left(\beta_{l}=1, \beta_{b}=0.6\right)$ : (a) moment load, (b) force load.

side and loaded at the free end, the following four boundary conditions and transformations can be applied for numerical solution:

$\kappa(L)=\frac{M}{E I_{\zeta}}, \theta(0)=0, u_{\xi}(0)=0, u_{\eta}(0)=0$.

This boundary value problem is solved approximately with MATLAB by means of a classical four-step Runge-Kutta method which is implemented in the used ode45 function. Based on the calculated deflected state due to the applied load in point $P_{2}^{\prime}$ the both displacements $u_{\xi}$ and $u_{\eta}$, and the bend- ing angle $\theta$ can be determined for every point $s$. We analysed the three results in point $P_{1}^{\prime}$, for example. Further, the bending angle $\theta(L)$ corresponds to the rotation angle $\varphi$ of the flexure hinge and it equals nearly exactly the bending angle in point $P_{1}^{\prime}$ too.

The following analytical characterization for the rotational stiffness, the strain, and the rotational precision is exemplified for the hard aluminium alloy EN AW 7075 with a Young's modulus of $E=72 \mathrm{GPa}$ and Poisson's ratio of $v=0.33$, which is widely used for precision engineering applications because of the high admissible elastic strain up to 


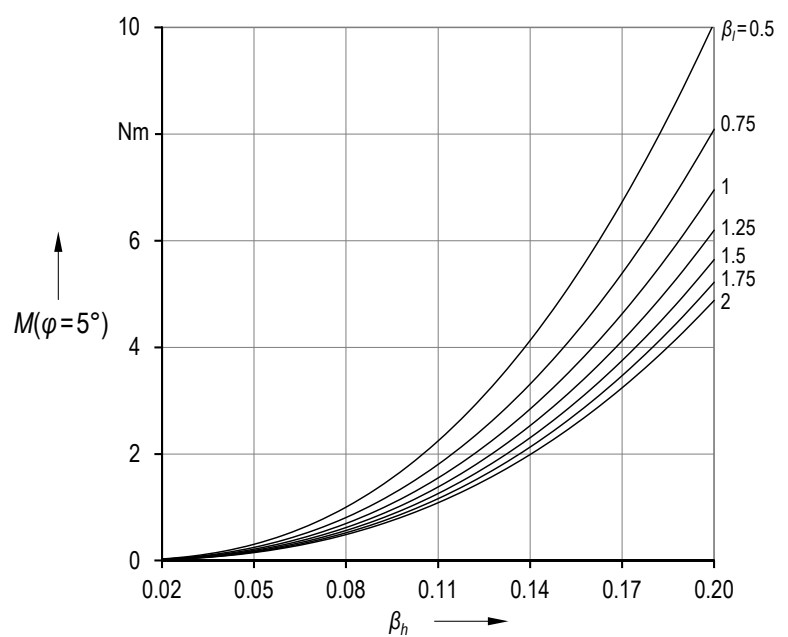

(a)

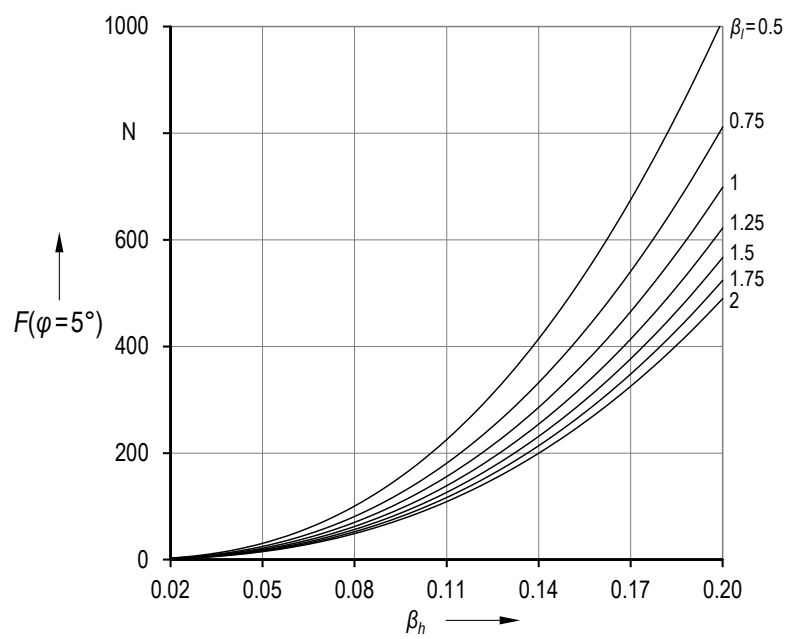

(b)

Figure 11. Required load for a discrete angular deflection - analytical results for a semi-circular flexure hinge at $\varphi=5^{\circ}$ in dependence of $\beta_{l}$ and $\beta_{h}\left(\beta_{b}=0.6\right)$ : (a) moment load, (b) force load.

$0.5 \%$. As assumption a typical hinge height of $H=10 \mathrm{~mm}$ is chosen. According to this, appropriate values of the geometric hinge parameters $\beta_{l}, \beta_{h}$, and $\beta_{b}$ are used to investigate the influence of these design parameters.

\subsection{Rotational stiffness}

As rotational stiffness of a flexure hinge in this paper the $M(\varphi)$ respectively $F(\varphi)$ characteristic is considered in dependence of the load case. The influence of the hinge contour on the rotational stiffness is exemplarily shown in Fig. 8 for hinge dimensions, which are appropriate in precision engineering applications.

The principal load-angle-behaviour is almost linear, which leads to a constant stiffness for the regarded small angular deflections up to $5^{\circ}$. The qualitative correlation and quantitative characteristic between the four hinge contours is similar for both loads. The following order can be generalized from the lowest to the highest stiffness: The corner-filleted contour, the polynomial 4th-order contour, the elliptical contour, and the semi-circular contour. A flexure hinge with a semicircular contour is six times stiffer than with a corner-filleted contour for the regarded basic hinge dimensions.

The influence of the basic hinge dimensions on the rotational stiffness is investigated for all hinge contours. The influence of $\beta_{l}$ is shown in Fig. 9 and the influence of $\beta_{h}$ is shown in Fig. 10 exemplarily for a flexure hinge with a semicircular contour, since they are state of the art. The following results can be generalized over all four flexure hinge contours:

- The rotational stiffness increases with a decreasing hinge length ratio $\beta_{l}$.
- The rotational stiffness increases with an increasing hinge height ratio $\beta_{h}$.

- The rotational stiffness increases with an increasing hinge width ratio $\beta_{b}$.

- The characteristic is qualitative and quantitative similar for both load cases.

Because flexure hinges are used in compliant mechanisms, the load characteristic for a discrete angular deflection is interesting too. The resulting loads $M$ and $F$ for a given angle $\varphi$ at the free end can be calculated using a further boundary condition $(\theta(L)=\varphi)$. Regarding the load for the example of a typical rotation angle of $\varphi=5^{\circ}$. it is obvious, that $\beta_{h}$ has a stronger influence on the rotational stiffness than $\beta_{l}$ independent form the load (see Fig. 11). Furthermore, if the rotation angle is given, any results can be calculated and used for design graphs to determine the required load for given hinge dimensions or vice versa.

\subsection{Strain distribution and maximal angular deflection}

In this section, additionally the bending stress is analysed after linear beam theory to characterize the maximum stress of the entire flexure hinge for a given deflection as a result of the moment or force load:

$\left.\sigma_{b}(\xi)\right|_{\eta_{\max }}=\left|\frac{M_{b}(\xi)}{w_{b}(\xi)}\right|$

According to the used theory, the maximum bending stress always results at the outer fibre for the maximum coordinate of $\eta$, which corresponds to the flexure hinge contour function. To characterize the stress of a flexure hinge, in literature mostly contour-dependent stress concentration fac- 


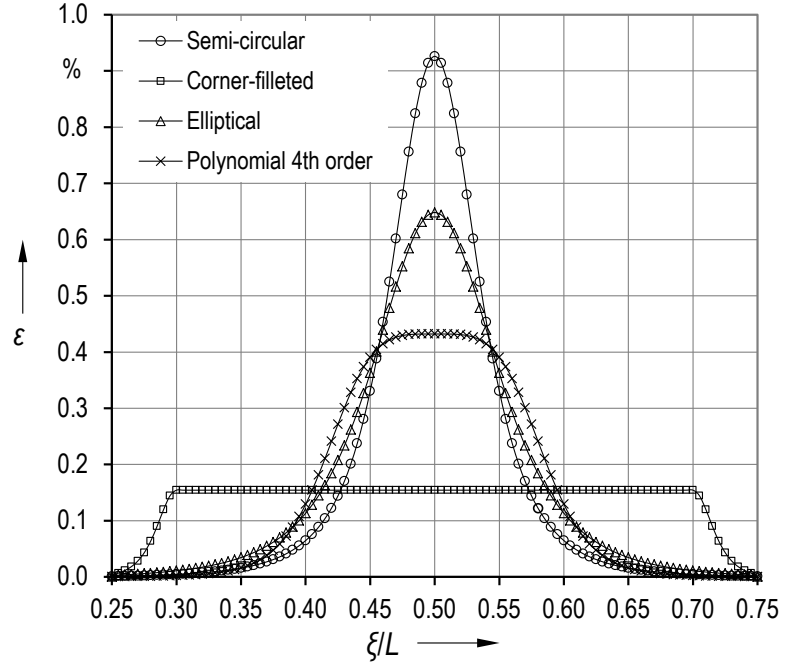

(a)

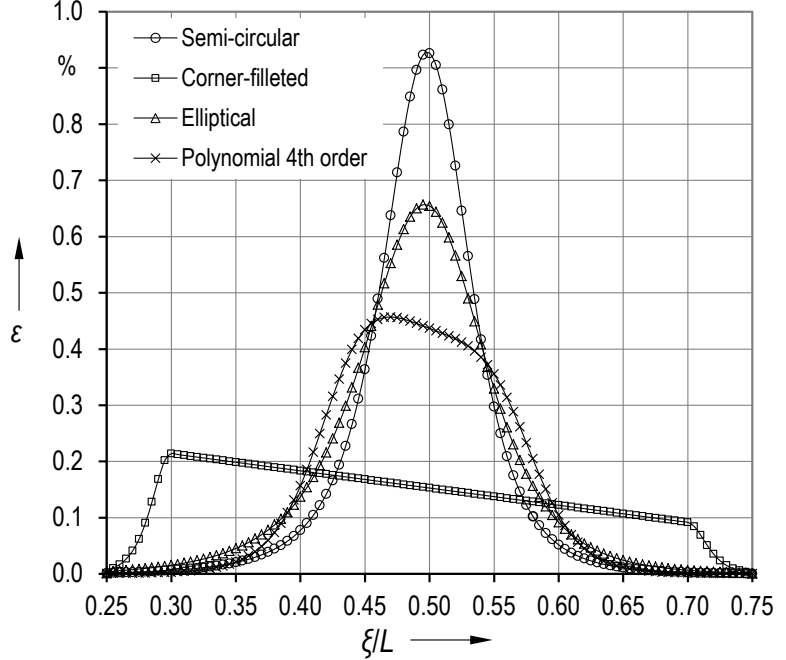

(b)

Figure 12. Analytical results for the strain distribution of a flexure hinge with various contours along $h_{\mathrm{n}}(\xi)$ at $\varphi=5^{\circ}\left(\beta_{l}=1, \beta_{h}=0.03\right)$ : (a) moment load, (b) force load

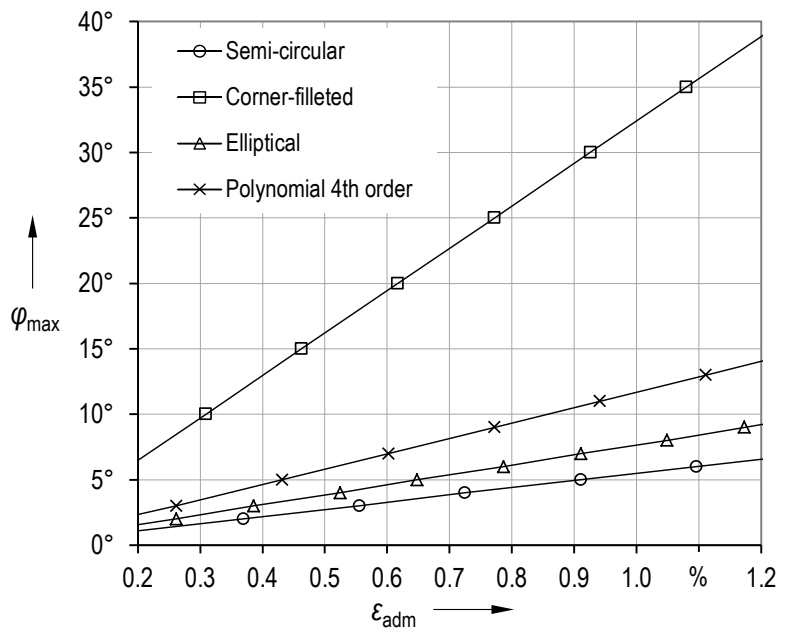

(a)

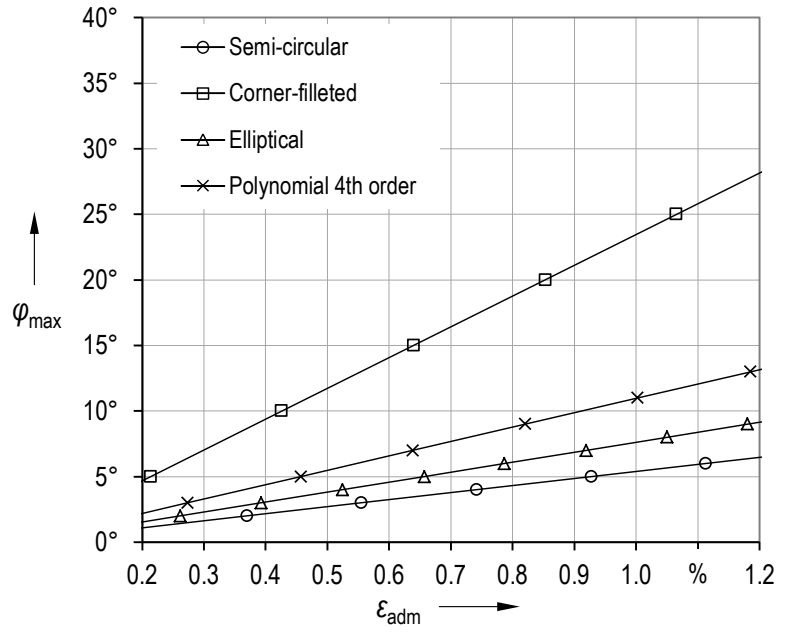

(b)

Figure 13. Analytical results for the maximal angular deflection $\varphi_{\max }$ of a flexure hinge with various contours in dependence of the admissible elastic strain $\varepsilon_{\mathrm{adm}}\left(\beta_{l}=1, \beta_{h}=0.03\right)$ : (a) moment load, (b) force load.

tors are considered (Chen et al., 2014), with which the increase of stress due to a contour variation is always estimated compared to a flexure hinge with the constant thickness $h$ (nominal stress of minimum cross-section). In this paper, the contour-dependent stress is directly determined by considering the exact contour function of each flexure hinge. With the elastic section modulus for a rectangular cross-section and according to elastic deformation with a linear relation between stress and strain, the material independent strain is considered below. Thus, in dependence of the load case the contour-dependent strain is given by Eq. (15) for a moment load and by Eq. (16) for a transverse force load.

$$
\begin{aligned}
\varepsilon(\xi) & =\frac{6 M}{E b h_{\mathrm{n}}^{2}(\xi)} \\
\varepsilon(\xi) & =\frac{6(L-\xi) F}{E b h_{\mathrm{n}}^{2}(\xi)}
\end{aligned}
$$

Now, taken into account a discrete angular deflection, we obtain the strain distribution along the outer fibre in dependence of the used contour function $h_{\mathrm{n}}$, as shown in Fig. 12 for both load cases and comparable hinge dimensions. The required input loads $M$ and $F$ are taken from Sect. 3.1. According 
to the theory, the maximum strain is independent from the width $b$ for a given deflection.

Among the four regarded contours, the semi-circular contour always leads to the highest strain values. In this case, the maximum admissible elastic strain would be exceeded for the aluminium material AW 7075. According to the following order - of using the elliptical, the polynomial, and the corner-filleted contour - the maximum strain value can be reduced.

For a moment load it is obvious, that the maximum strain occurs in the hinge centre in general. In contrast to this, the critical coordinate $\xi_{\text {crit }}$ depends on the flexure hinge contour for a force load. While the maximum strain approximately occurs in the hinge centre for semi-circular and elliptical contours too, the critical point $\xi_{\text {crit }}$ moves in direction of the clamped hinge side the more the flexure hinge contour takes the form of a simple beam, e.g. the corner-filleted contour.

Furthermore, regarding a concrete application in a compliant mechanism, the admissible elastic strain $\varepsilon_{\mathrm{adm}}$ of the used material should be higher than the critical strain of each flexure hinge:

$\varepsilon_{\mathrm{adm}} \geq \varepsilon\left(\xi_{\text {crit }}\right)=\frac{6 M}{E b h_{\mathrm{n}}^{2}\left(\xi_{\text {crit }}\right)}$,

$\varepsilon_{\mathrm{adm}} \geq \varepsilon\left(\xi_{\text {crit }}\right)=\frac{6\left(L-\xi_{\text {crit }}\right) F}{E b h_{\mathrm{n}}^{2}\left(\xi_{\text {crit }}\right)}$.

Hence, the contour-dependent correlation of the materialgiven admissible elastic strain $\varepsilon_{\text {adm }}$ to the maximum allowable rotation angle $\varphi_{\max }$ is interesting. We obtain this correlation by stepwise increasing the load, determining the critical strain with a MATLAB procedure, and calculating the rotation angle according to the numerical solution of Eqs. (9) to (12). The resulting $\varphi_{\max }\left(\varepsilon_{\mathrm{adm}}\right)$ correlation is shown in Fig. 13 for both load cases. Of course, for the practical application it is common to use a safety factor in addition. But this factor can be simply included in the admissible strain value too.

Because the maximum strain value limits the deflection, the maximum rotation angle of a flexure hinge always is possible with a corner-filleted contour, while a semi-circular contour leads to the lowest possible angles. The rotation angle of corner-filleted contour is more than five respectively four times higher than of a semi-circular contour. Especially for flexure hinges made from plastic, with admissible strain values higher than $1 \%$, a large angular deflection can be realized in dependence of the chosen minimal height $h$. According to the same position of $\xi_{\text {crit }}$, the $\varphi_{\max }\left(\varepsilon_{\text {adm }}\right)$ curve of a semi-circular and elliptical contour shows a qualitative and quantitative similar behaviour for both load cases. Due to a higher maximum strain, the maximum angular deflection decreases for a force load, at most for a corner-filleted contour.

\subsection{Rotational precision}

In particular in precision engineering, the rotational precision of a flexure hinge is a very important performance criterion for the kinematic behaviour of a compliant mechanism. Because of the serial connection of several flexure hinges in the kinematic chain, the rotational axis shift $v$ of a single flexure hinge (cf. Fig. 7) can influence the path deviation of a coupler point of the compliant mechanism compared to the rigid-body mechanism. As rotational precision of a flexure hinge in this paper the rotational axis shift-angle characteristic $v(\varphi)$ is considered in dependence of the load case.

Since there is no stationary rotation axis, the rotational axis shift $v$ has to be determined model-based. Therefore, in literature four different approaches to define the axis of rotation of a notch flexure hinge are mainly suggested, which lead to a different direction and absolute value of the axis shift (Linß et al., 2011a): Mostly, the shift respectively offset $u\left(s=\frac{L}{2}\right)$ of the centre point of the flexure hinge at the neutral axis is calculated on the basis of the deformed state (e.g. Lobontiu, 2003; Chen et al., 2005; Tian et al., 2010), whereas the kinematic behaviour is not considered. A second approach considers the intersection of the tangents at the neutral axis in the two points $O$ and $P_{1}^{\prime}$, why the axis shift can only be calculated in $\xi$ direction (e.g. Horie et al., 1997; Smith, 2000). Thirdly, the fixed centrode is calculated with the help of a geometrical approximation method (e.g. Dirksen and Lammering, 2011; Palmieri et al., 2012), what leads to high numerical effort since the accuracy here depends on the given step size and the load levels (infinitesimal analysis). Fourthly, a rigid-body model based approach considers the guiding of the initial centre point with a constant distance of the half hinge length during the motion (fixed centre approach, e.g. Tseytlin, 2002; Zelenika et al., 2009). As comparative investigations show, the latter approach is suitable regarding a direct and accurate modelling of the rotational axis of a flexure hinge (Lin $\beta$ et al., 2011a). Therefore, the fixed centre approach is chosen to investigate the rotational precision in this paper (cf. Fig. 7).

In this case, the absolute value of the rotational axis shift $v$, based on the hinge centre point in the initial position, equals the circular path deviation of any regarded point on the loaded segment of the flexure hinge:

$$
\begin{aligned}
v & =\sqrt{\left(\xi_{C^{\prime}}-\frac{L}{2}\right)^{2}+\eta_{C^{\prime}}^{2}} \\
& =\sqrt{\left(u_{\xi}-\frac{l}{2} \cos \varphi+\frac{l}{2}\right)^{2}+\left(u_{\eta}-\frac{l}{2} \sin \varphi\right)^{2}} .
\end{aligned}
$$

To determine the axis shift, the deformation $u$ of one arbitrary point at the free end and the rotation angle $\varphi$ must be known only. In this case, we used the deformation $u\left(\frac{L}{2}+\frac{l}{2}\right)$ in Point $P_{1}^{\prime}$. Thus, the simultaneous investigation based on analytical, experimental and simulative methods is possible. Due to the used beam theory, the variation of the hinge contour and the $\xi$-displacement of the free end are considered, which has a non-negligible influence on the axis shift too. Furthermore, for a force load it is known, that the direction of the shift is 


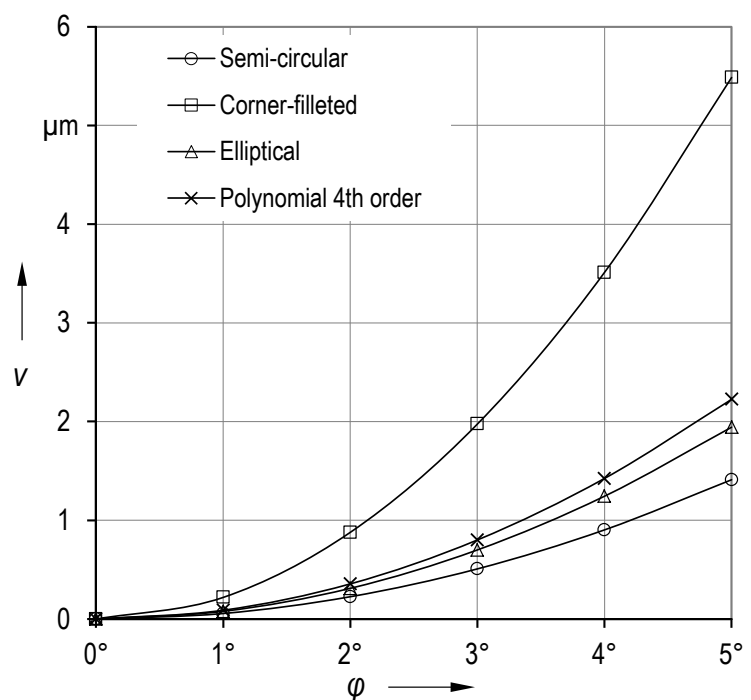

(a)

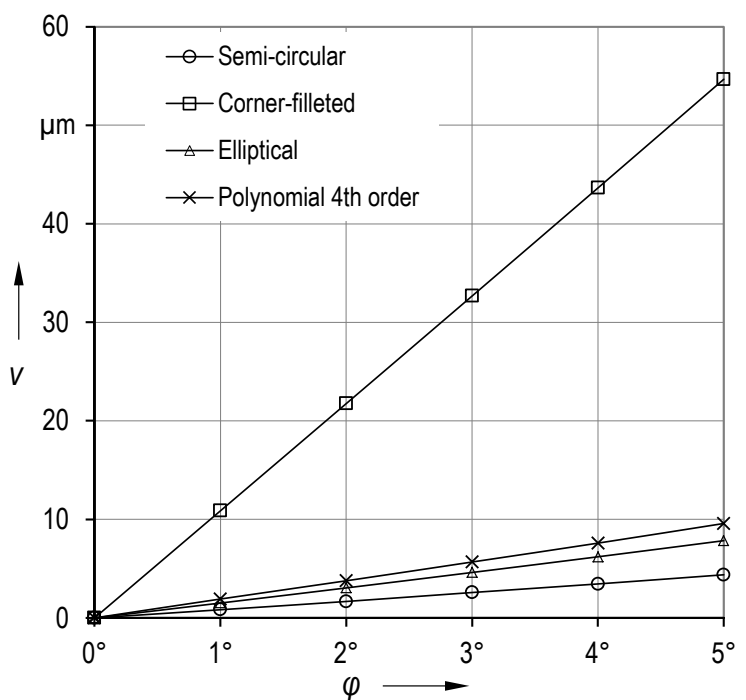

(b)

Figure 14. Influence of the hinge contour - analytical results for the rotational precision of a flexure hinge with various contours $\left(\beta_{l}=1\right.$, $\beta_{h}=0.03$ ): (a) moment load, (b) force load.

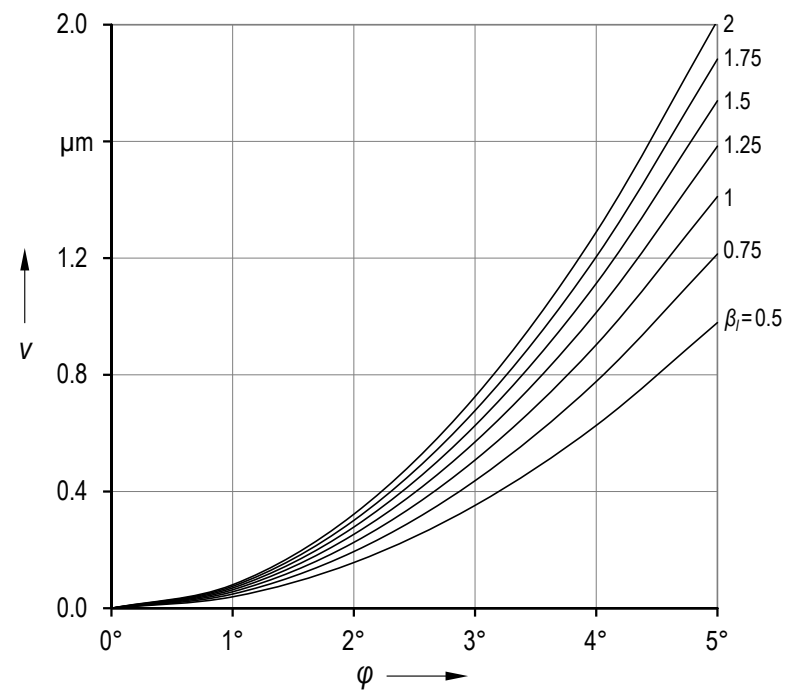

(a)

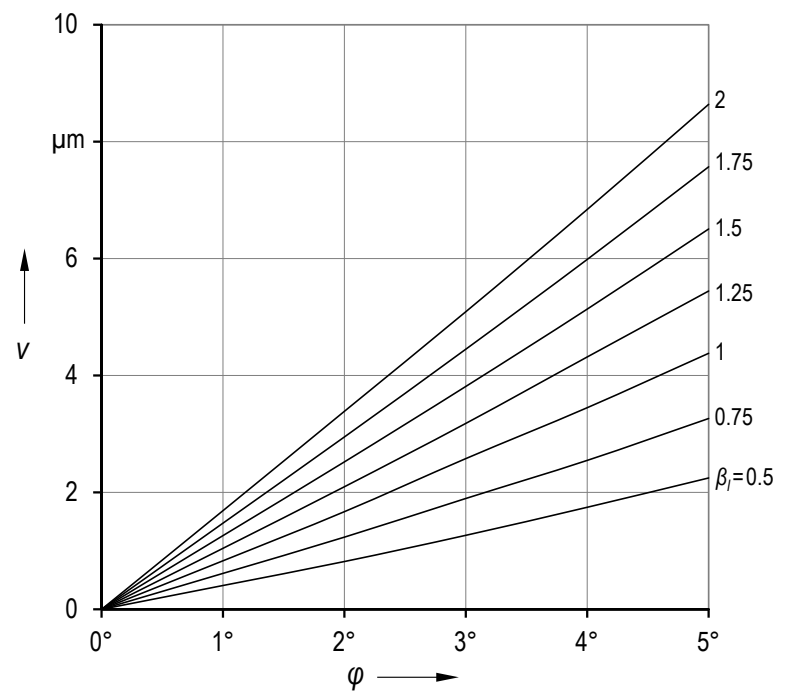

(b)

Figure 15. Influence of the hinge dimensions - analytical results for the influence of $\beta_{l}$ on the rotational precision of a semi-circular flexure hinge ( $\left.\beta_{h}=0.03\right)$ : (a) moment load, (b) force load.

dominant according to the force direction. However, in this paper the absolute value of the rotational axis shift is in the focus of investigations.

The influence of the hinge contour on the rotational precision is shown in Fig. 14. The qualitative axis shift-anglebehaviour is non-linear for a moment load and almost linear for a force load. Furthermore, the load case has an influence on the absolute value: Independent from the hinge contour, a transverse force leads to a significant larger axis shift than a moment load for an equal angle $\varphi$.
The hinge contour has a strong influence on the axis shift, which can be in the range of several micrometres up to the millimetre range in dependence of the basic dimensions $\beta_{l}$ and especially $\beta_{h}$. With regard to a high rotational precision respective a small axis shift the following order can be generalized for thin hinges: The semi-circular contour, the elliptical contour or polynomial 4th order contour, and the cornerfilleted contour. A flexure hinge with a semi-circular contour can be more than 30 times more precise than with a cornerfilleted contour (e.g. for $\beta_{l}=1, \beta_{h}=0.01$, and a force load). 


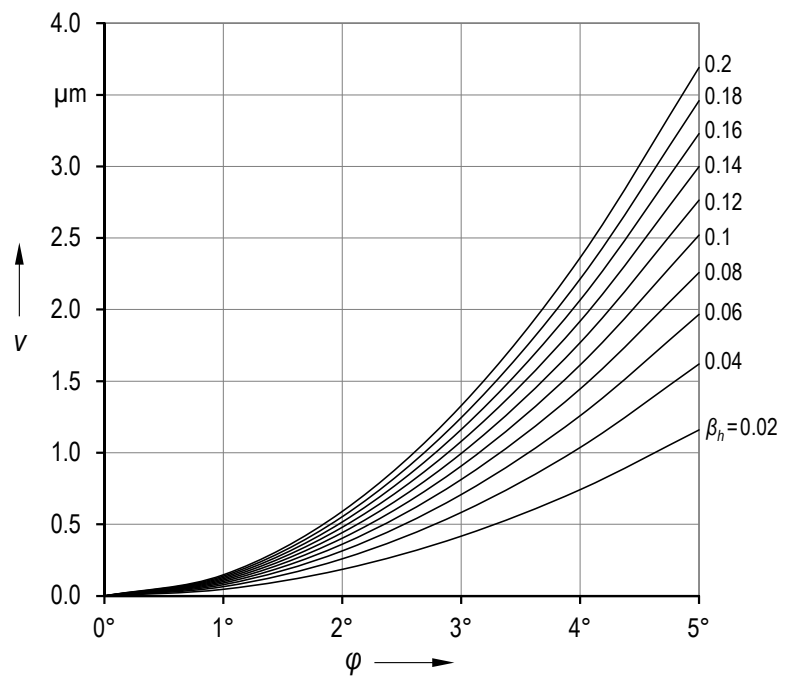

(a)

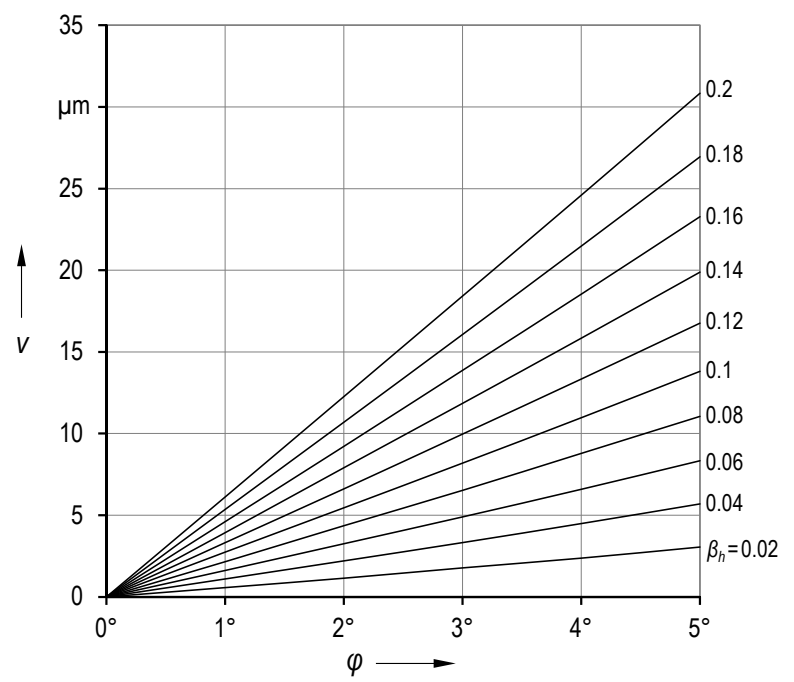

(b)

Figure 16. Influence of the hinge dimensions - analytical results for the influence of $\beta_{h}$ on the rotational precision of a semi-circular flexure hinge $\left(\beta_{l}=1\right)$ : (a) moment load, (b) force load.

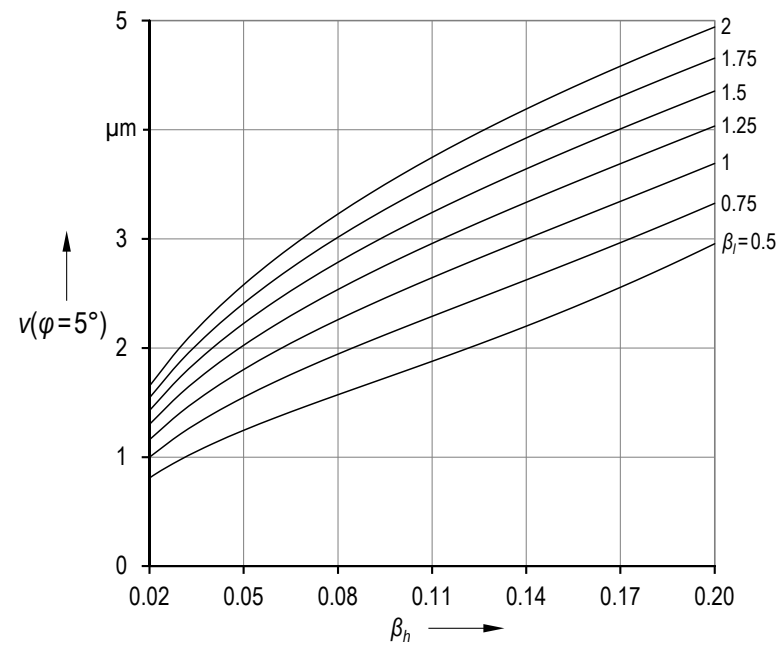

(a)

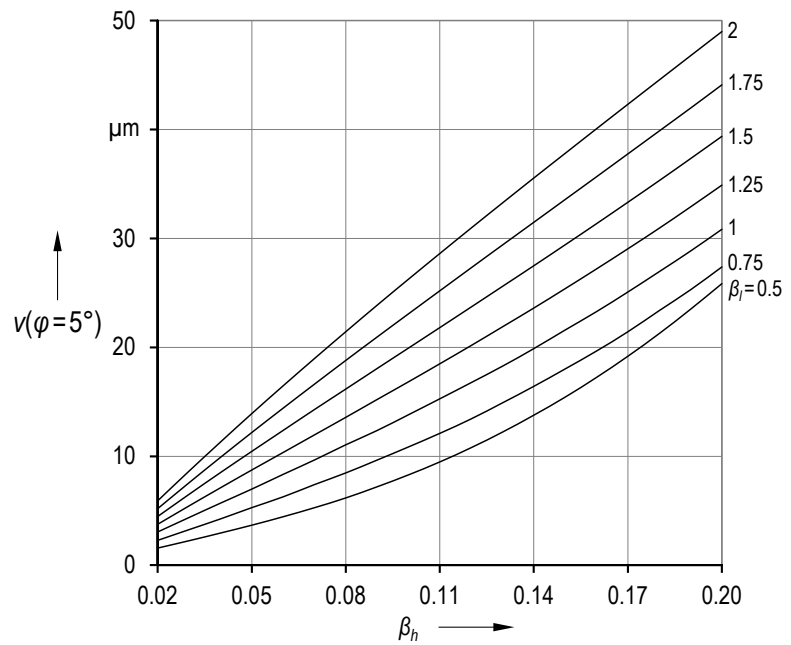

(b)

Figure 17. Rotational axis shift for a discrete angular deflection - analytical results for a semi-circular flexure hinge at $\varphi=5^{\circ}$ in dependence of $\beta_{l}$ and $\beta_{h}$ : (a) moment load, (b) force load.

But especially for short and thick hinges, a corner-filleted contour is exactly as precise as a semi-circular contour (e.g. for $\beta_{l}=0.5, \beta_{h}=0.2$, and a force load). This is a novel result and it has been confirmed for the use of flexure hinges in compliant mechanisms too (Linß et al., 2014).

The influence of the basic hinge dimensions $\beta_{l}$ and $\beta_{h}$ on the rotational precision is shown in Figs. 15 and 16 for a flexure hinge with a semi-circular contour. The following results can be generalized over all four flexure hinge contours:

- The rotational precision increases (the axis shift decreases) with a decreasing hinge length ratio $\beta_{l}$.
- The rotational precision increases with an increasing hinge height ratio $\beta_{h}$.

- The rotational precision and the kinematic behaviour are independent of the hinge width ratio $\beta_{b}$.

- The characteristic is qualitative and quantitative different for both load cases.

- The influence of the hinge contour decreases with an increasing hinge height ratio $\beta_{h}$, in particular for thick hinges. 
Finally, the rotational axis shift for a discrete angular deflection of $\varphi=5^{\circ}$ is shown in Fig. 17 in dependence of $\beta_{l}$ and $\beta_{h}$ for both load cases. Hence, if the rotation angle is given, any results can be calculated and used for design graphs to predict the rotational precision of a flexure hinge for given basic dimensions or to determine the dimensions in order to realize required specifications.

\section{Design equations for the flexure hinges with various notch geometries}

In this section, novel general design equations for the rotational stiffness, the maximal angular elastic deflection, and the rotational precision of a notch flexure hinge in dependence of the load case are derived based on the analytical results. The approach and the method are described briefly and the developed design equations are presented together with the contour-specific coefficients.

In literature, design equations exist in particular for the rotational stiffness of a flexure hinge with a semi-circular, corner-filleted or elliptical contour (e.g. Paros and Weisbord, 1965; Tseytlin, 2002; Wu and Zhou, 2002; Lobontiu, 2003; Chen et al., 2008, 2011; Meng et al., 2013). Design equations for calculating the contour-dependent maximal angular elastic deflection are not state of the art, because the few existing design equations consider solely the stress (e.g. Chen et al., 2014) or the correlation between the load and the strain (Tres, 1995; Kunz, 2007; Dirksen, 2013), but not explicit the rotation angle. Especially regarding the rotational precision, the presented design equations are limited due to the use of standard hinge contours and a different approach to model the axis of rotation. In literature, closed-form equations are mainly presented for the approach of the offset of the hinge centre point (e.g. Lobontiu, 2003; Chen et al., 2009; Hu et al., 2012; Li et al., 2013). Rarely, empirical design equations based on FEM simulation results are suggested for semicircular (Yong et al., 2008) and corner-filleted (Meng et al., 2013) flexure hinges.

However, all suggested design equations are characterized by a long expression and complex structural form, and they are only valid for a special group of flexure hinge contours. Simple and concise analytically derived design equations, whose principal structural form is independent from the notch geometry, are not known to the authors.

To develop the novel design equations, first the analytical results for the rotational stiffness and rotational precision of a flexure hinge are calculated, as in Sect. 3 described, for the following parameters and ranges of variation:

- four flexure hinge contours (semi-circular, cornerfilleted, elliptical and bi-quadratic polynomial contour);

- three basic hinge dimensions of $\beta_{l}$ (in the range of 0.5 to 2 in steps of 0.25$), \beta_{h}$ (0.01 to 0.2 in steps of 0.01 ) and $\beta_{b}$ (0.1 to 1 in steps of 0.1 ;
- the angular deflection of $\varphi \leq 5^{\circ}$ (in steps of $1^{\circ}$ );

- the load case of a moment load or a transverse force load close to the hinge centre at $L=2 H=20 \mathrm{~mm}$ (free end).

Second, with the help of MATLAB the contour-specific coefficients of a power function are determined based on a fitting procedure in order to realize the smallest maximum error over all calculated result points. The approach is explained in Sect. 4.1 exemplarily for the rotational stiffness and the moment load case. The remaining design equations for the contour-dependent maximal angular deflection are obtained by conversion and the additional introduction of a correction factor for considering the location of the critical strain. Finally, the rounded coefficients (two digits) are determined again for a reduced parameter range for $\beta_{l}\left(0.5 \leq \beta_{l} \leq 1.5\right)$ and $\beta_{h}\left(0.03 \leq \beta_{h} \leq 0.1\right)$ in order to improve the accuracy of the developed design equations (see Table 1). According to the theory, the accuracy of the results is independent of the parameter range for $\beta_{b}$. Depending on the contour, the relative root mean squared error $e_{\text {rms }}$ over all parameter combinations is in the range of less than $2 \%$ to less than $16 \%$ compared to the analytical solution.

\subsection{Rotational stiffness}

Based on the numerically calculated analytical results, a power function is used to express the functional load-anglecorrelation $M(\varphi)$ respectively $F(\varphi)$ in dependence of the relevant geometric parameters over all parameter ranges. The used power function for the moment load for example has the following initial form:

$$
\frac{M}{\varphi}=k_{1} E \beta_{b} \beta_{l}^{\left(k_{2}\right)} \beta_{h}^{\left(k_{3}\right)} H^{\left(1+k_{2}+k_{3}\right)} .
$$

A power function with three coefficients $k_{1}, k_{2}$ and $k_{3}$ was used at first, to consider the different characteristic for each flexure hinge contour. We have chosen the power function because its form is suitable to express the principle dependence of the basic geometric parameters, which is strictly monotonic increasing. Furthermore, the power function of fers great shape variability with a minimal number of coefficients. Because the correlation of $M$ and $b$ is exactly proportional, coefficients are only considered for the geometric parameters $l$ and $h$.

Following the dimensional analysis theory, we consider the geometric hinge parameters according to the defined dimensionless ratios $\beta_{l}, \beta_{h}$, and $\beta_{b}$. Due to this, the only remaining parameter with a length unit is the total height $H$. Thus, a simple scaling of the whole flexure hinge is possible in dependence of the chosen value of $H$.

To obtain the optimal coefficients of the non-linear power function a curve fit was realized based on the Nelder-Mead simplex algorithm for each hinge contour. This optimization 


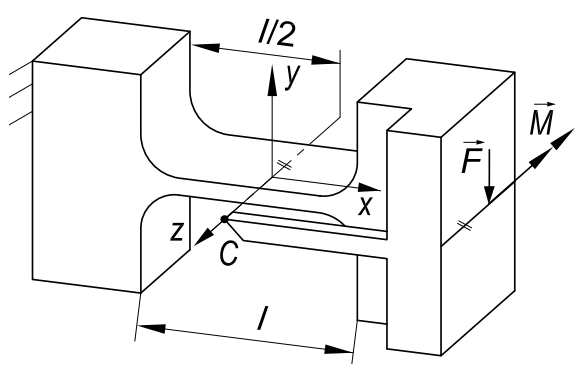

(a)

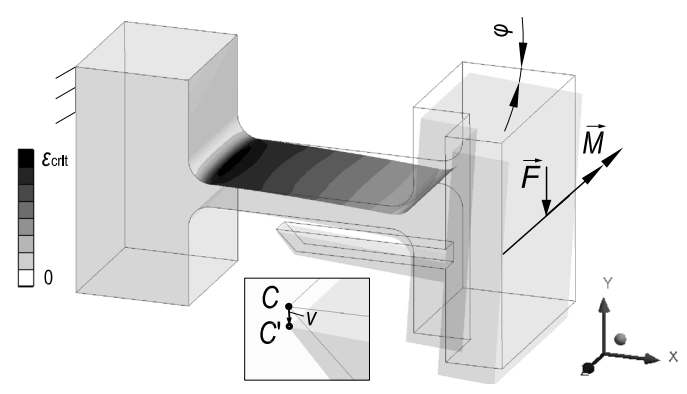

(b)

Figure 18. FEM-based characterization of a flexure hinge: (a) CAD Model, (b) FEM model with initial and deformed hinge.

Table 1. Coefficients for design Eqs. (21)-(26) in dependence of the flexure hinge contour, valid for appropriate hinge dimensions $\left(0.5 \leq \beta_{l} \leq 1.5,0.03 \leq \beta_{h} \leq 0.1, \beta_{b}\right.$ arbitrary) and an angular deflection $\varphi \leq 5^{\circ}$.

\begin{tabular}{|c|c|c|c|c|c|c|c|c|c|c|}
\hline Hinge contour & $k_{M 1}\left[10^{-3}\right]$ & $k_{M 2}$ & $k_{F 1}\left[10^{-2}\right]$ & $k_{F 2}$ & $k_{\text {crit }}$ & $k_{v M 1}\left[10^{-3}\right]$ & $k_{v M 2}$ & $k_{v F 1}\left[10^{-2}\right]$ & $k_{v F 2}$ & $e_{\mathrm{rms}}[\%]$ \\
\hline Semi-circular & 107.9 & 0.52 & 10.55 & 0.51 & 0.5 & 99.85 & 0.52 & 19.12 & 0.94 & 4.64 \\
\hline Corner-filleted & 83.95 & 0.96 & 8.41 & 0.96 & $0.5-0.2 \beta_{l}$ & 85.76 & 0.95 & 9.2 & 1.89 & 2.08 \\
\hline Elliptical & 82.5 & 0.54 & 8.27 & 0.54 & 0.5 & 114.35 & 0.57 & 18.21 & 1.14 & 2.5 \\
\hline Bi-quadratic polynomial & 112.1 & 0.74 & 10.79 & 0.72 & $0.4 \beta_{l}^{(-0.08)} \beta_{h}^{(-0.04)}$ & 69.78 & 0.74 & 5.83 & 1.5 & 15.96 \\
\hline
\end{tabular}

has been implemented in MATLAB with the function fminsearch in order to minimize the maximum error of all calculated results of the design equation compared with each analytical result over the parameter range. Since a special correlation between the both coefficients $k_{2}$ and $k_{3}$ have become obvious during the investigations for each performance criterion, we express this with one combined coefficient below. This leads to a simple and short design equation with only two coefficients.

Hence, for a moment load we finally obtain the contourindependent design equation for the rotational stiffness of a flexure hinge as

$$
\frac{M}{\varphi}=k_{M 1} E \beta_{b} \beta_{l}^{\left(-k_{M 2}\right)} \beta_{h}^{\left(2+k_{M 2}\right)} H^{3},
$$

and taken into account a transverse force load we obtain the rotational stiffness as

$$
\frac{F}{\varphi}=k_{F 1} E \beta_{b} \beta_{l}^{\left(-k_{F 2}\right)} \beta_{h}^{\left(2+k_{F 2}\right)} H^{2} .
$$

The resulting contour-dependent coefficients for all design equations are given in Table 1.

\subsection{Maximal angular deflection}

To obtain the design equations for the $\varphi_{\max }\left(\varepsilon_{\mathrm{adm}}\right)$ correlation in dependence of the load case, the load $M$ (represented in Eq. 21) and the load $F$ (Eq. 22) are used in Eqs. (17) and (18). Next, the critical location $\xi_{\text {crit }}$ for the maximum strain at the outer fibre and the hinge height $h_{\mathrm{n}}\left(\xi_{\text {crit }}\right)$ for this location have to be considered in dependence of the load case.
For a moment load, the maximum strain occurs contourindependent in the hinge centre. Therefore, the critical hinge height $h_{\mathrm{n}}\left(\xi_{\text {crit }}\right)$ always equals the minimum hinge height $h$. If $h$ is expressed by $\beta_{h}$, this leads to the following design equation for the maximal angular deflection due to a moment load:

$\left|\varphi_{\max }\right|=\frac{\varepsilon_{\mathrm{adm}}}{6 k_{M 1}}\left(\frac{\beta_{l}}{\beta_{h}}\right)^{k_{M 2}}$.

For a transverse force load, the critical location depends on the hinge contour. For this reason, an additional dimensionless correction factor $k_{\text {crit }}$ for the deviation to the hinge centre at $\xi=\frac{L}{2}$ is introduced in this case, and the maximum length $L$ is eliminated. This leads to the following design equation for the maximal angular deflection due to a transverse force load:

$\left|\varphi_{\max }\right|=\frac{\varepsilon_{\mathrm{adm}}}{12\left(1-k_{\mathrm{crit}}\right) k_{F 1}}\left(\frac{\beta_{l}}{\beta_{h}}\right)^{k_{F 2}}$.

According to the results in Sect. 3.2 (cf. Fig. 12b) it is assumed, that the maximum strain occurs approximately in the hinge centre for a semi-circular and an elliptical contour, what leads to $k_{\text {crit }}=0.5$. The correction factor for the investigated corner-filleted contour with an optimal fillet radius can be expressed by the geometric parameter $\beta_{l}$ in general (see Table 1). Thus, in all three cases, the relevant critical hinge height $h_{\mathrm{n}}\left(\xi_{\text {crit }}\right)$ equals the minimum hinge height $h$ too. To determine the correction factor for the polynomial contour of 4th order, a power function with three constants was fitted with the function fminsearch in MATLAB too, based on the calculated results for the locations $\xi_{\text {crit }}$ over the parameter range. 


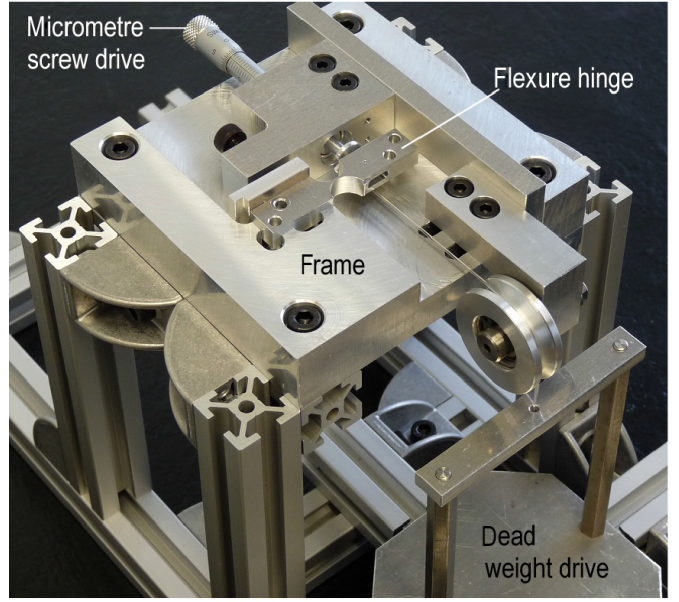

(a)

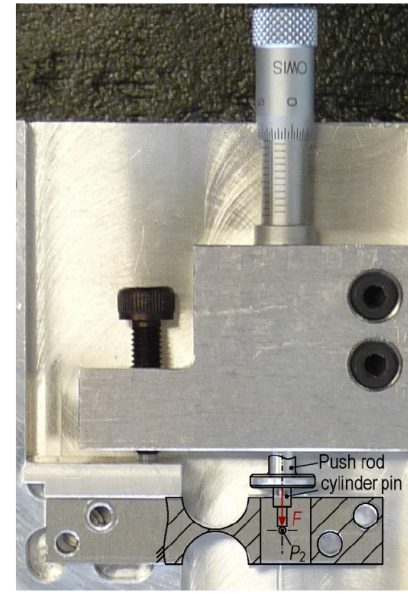

(b)
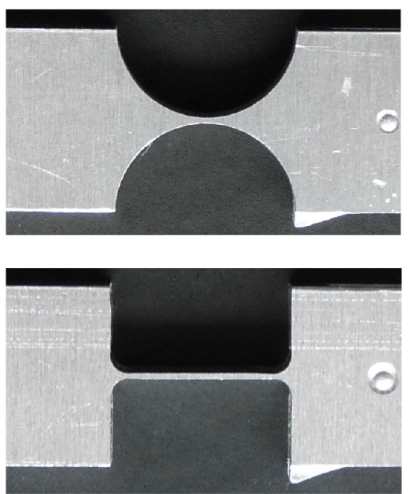

(c)

Figure 19. Experimental characterization of a force loaded flexure hinge: (a) experimental setup, (b) displacement or force driven load in $P_{2}$, (c) test specimens with a semi-circular and corner-fileted hinge contour $\left(\beta_{l}=1, \beta_{h}=0.03, \beta_{b}=0.6\right.$, aluminium AW 7075).

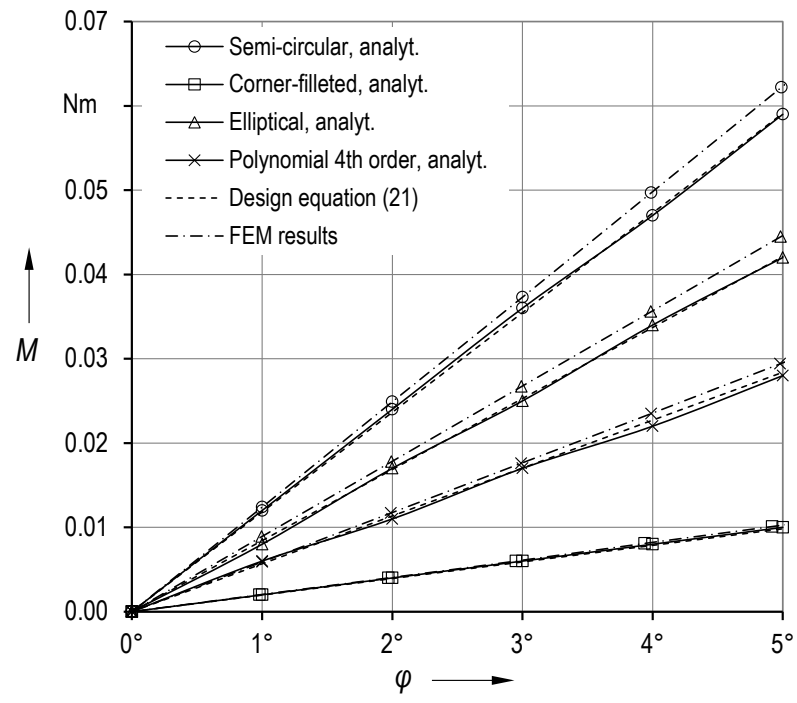

(a)

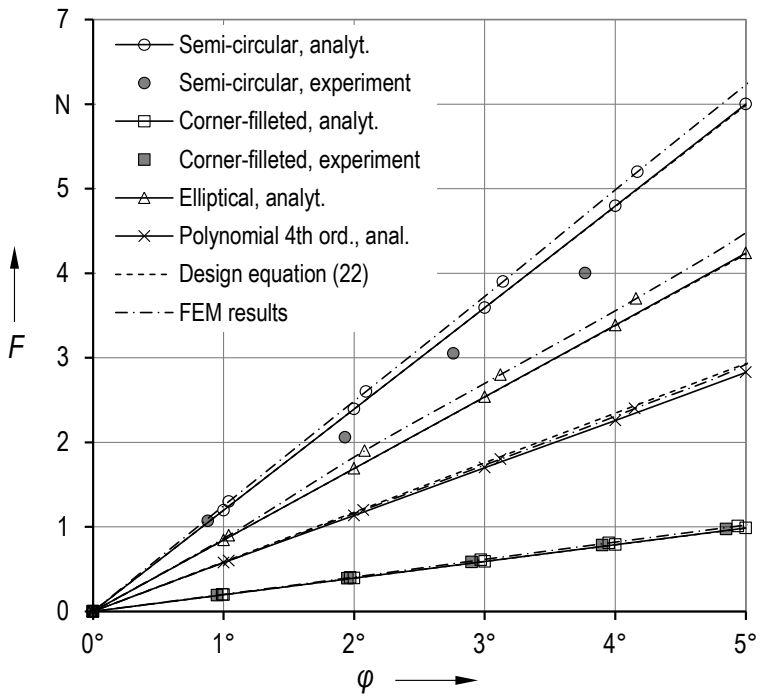

(b)

Figure 20. Comparison of results for the rotational stiffness of a flexure hinge with various contours $\left(\beta_{l}=1, \beta_{h}=0.03, \beta_{b}=0.6\right)$ : (a) moment load, (b) force load.

\subsection{Rotational precision}

The design equations for the $v(\varphi)$ characteristic were developed based on the power function, as already described in Sect. 4.1. According to the analytical results (cf. Sect. 3.3) it is further assumed that $v$ correlates with $\varphi^{2}$ for a moment load and with $\varphi$ for a force load.

Hence, for a moment load we obtain the contourindependent design equation for the rotational precision of a flexure hinge as:

$\frac{v}{\varphi^{2}}=k_{v M 1} \beta_{l}^{k_{v M 2}} \beta_{h}^{\left(1-k_{v M 2}\right)} H$ and taken into account a transverse force load we obtain the rotational precision as

$\frac{v}{\varphi}=k_{v F 1} \beta_{l}^{k_{v F 2}} \beta_{h}{ }^{\left(2-k_{v F 2}\right)} H$.

\section{Discussion of results}

In this section, the design equation results are compared with the analytical results, and with further results based on FEM simulations and experimental tests of selected flexure hinge contours. The FEM model and the experimental setup are described briefly. Then, the results are discussed for appropriate hinge dimensions. 


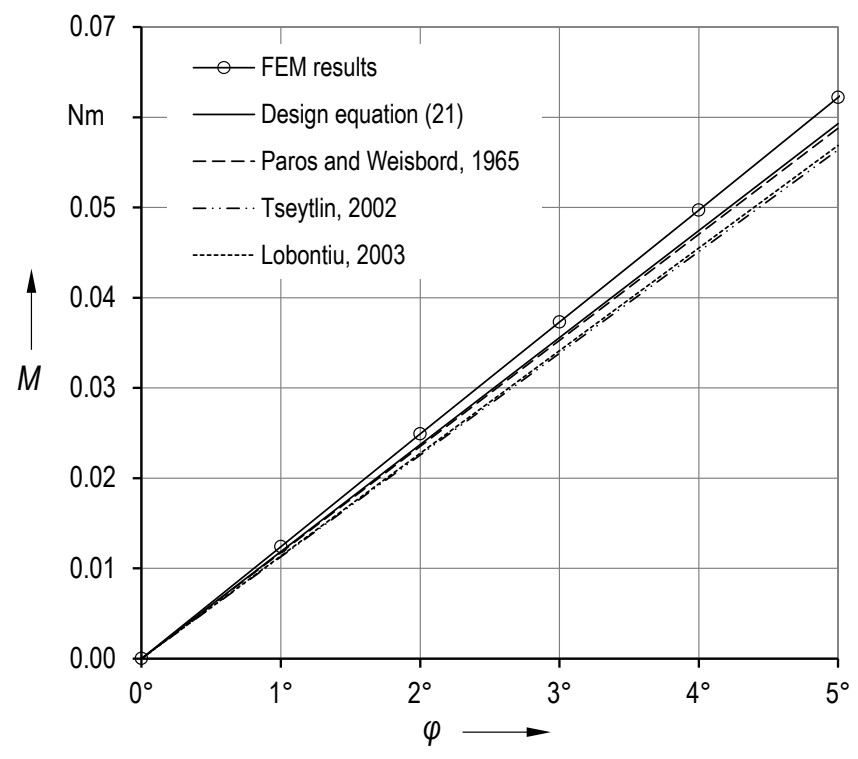

Figure 21. Comparison of results with existing design equations for the rotational stiffness of a moment loaded semi-circular flexure hinge $\left(\beta_{l}=1, \beta_{h}=0.03, \beta_{b}=0.6\right)$.

\subsection{FEM-based characterization}

For the FEM-based simulation of the flexure hinges ANSYS Workbench 16.2 is used. The CAD model and the FEM model are shown in Fig. 18. The CAD model includes an additional part to realize the direct determination of the rotational axis $C$ according to the chosen fixed centre approach (cf. Fig. 7). In the FEM model one end of the flexure hinge is fixed and the free end is loaded with a moment or transverse force load at the edge in point $P_{2}$. After determining the resulting rotation angle with two points of the free end, the three performance criteria, the rotational stiffness $M(\varphi)$ respectively $F(\varphi)$, the maximal angular deflection $\varphi_{\max }\left(\varepsilon_{\mathrm{adm}}\right)$ and the rotational precision $v(\varphi)$ are analysed in dependence of the hinge contour for the investigated geometric parameters.

According to the literature, the flexure hinge is modelled as a solid structure (Zhang and Hu, 2009) with adjacent link segments (Zettl et al., 2005; Yong et al., 2008), like they are considered for the analytical characterization too. In the FEM simulation large deflection is considered due to nonlinear beam theory. Further assumptions are linear material behaviour for the used aluminium AW 7075 material (with the same parameters like in Sect. 3) and a comparable and fine discretisation of the hinge for all the different contours.

\subsection{Experimental characterization}

For the experimental investigation of the deflected state of the flexure hinge a coordinate-reading microscope (Carl Zeiss ZKM 01-250C) was used. The realized experimental setup with the clamped flexure hinge is shown in Fig. 19. The frame of the experimental setup is fixed to a $x y$ stage, which is synchronized with the microscope during the measurement. By the optical measurement of the coordinates of two points at the free end, the resulting rotation angle $\varphi$ and thus the calculated location of the rotational axis $C^{\prime}$ can be determined due to the given load.

Because the load case of an ideal moment cannot be realized due to the inherent rotational axis shift with the required accuracy, a transverse force is regarded only. In order to measure the rotational precision and the rotational stiffness, the force load can be implemented displacement driven (micrometre screw drive, Owis MS 6-12) or force driven (dead weight drive). The force load is transmitted from the input by a pushing rod and a cylindrical pin to a second orthogonal pin, which is fixed to the flexure hinge and which is coincident with the load acting point $P_{2}$. Because of the widest expected difference, as test specimens two flexure hinges with a semi-circular and a corner-fileted contour are investigated.

\subsection{Comparison of results for appropriate hinge dimensions}

For the comparison of the design equation results for various notch flexure hinges with the results of the analytical, FEM-based and experimental characterization, typical parameter values for the hinge dimensions $\left(\beta_{l}=1, \beta_{h}=0.03\right.$, $\left.\beta_{b}=0.6\right)$ are used. The results are shown in Fig. 20 for the rotational stiffness, in Fig. 22 for the maximal angular deflection, and in Fig. 23 for the rotational precision. Additionally, in Fig. 21 the deformation characteristic of a moment loaded semi-circular flexure hinge is compared with design equations from the literature and with the FEM simulation.

Generally, regarding all three performance criteria, the equation-based results are in good correlation with the analytical solution as well as with the FEM simulation and the measured results (qualitative as well as quantitative). Thus, the principle conclusions of Sect. 3 concerning the influence of the hinge contour and the geometric parameters on the rotational precision, the maximal angular deflection and the rotational precision have been confirmed by different methods. The little differences, especially to the FEM results, can be explained in particular with a more accurate modelling of a flexure hinge by means of FEM simulation. Regarding the experimental investigation, especially the measurement of the rotational axis shift in the micrometre range is challenging, but the principle absolute value could be confirmed for a flexure hinge with a semi-circular and a corner-filleted contour.

The comparison of results for the rotational stiffness of a moment loaded semi-circular flexure hinge with existing design equations from literature (cf. Fig. 21) and the FEM result shows, that Eq. (21) is accurate and very close to the simplified expression of Paros and Weisbord (1965). The used equations from the literature are mentioned in Eqs. (A1), (A2) and (A3). 


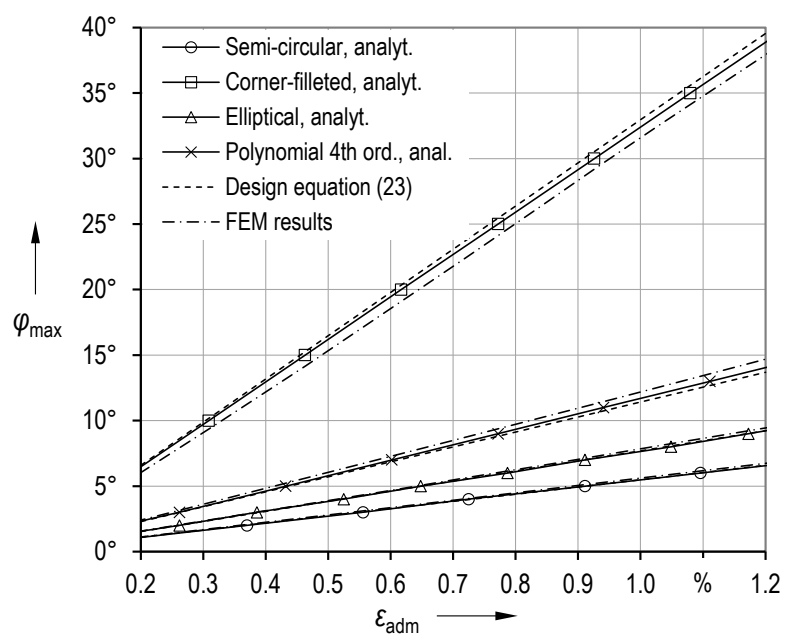

(a)

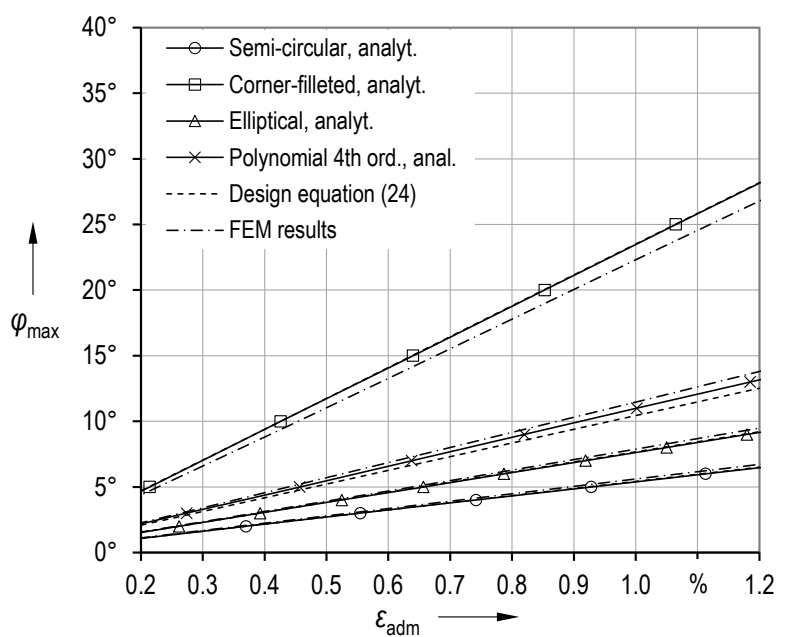

(b)

Figure 22. Comparison of results for the maximal angular deflection of a flexure hinge with various contours in dependence of the admissible material strain $\left(\beta_{l}=1, \beta_{h}=0.03\right)$ : (a) moment load, (b) force load.

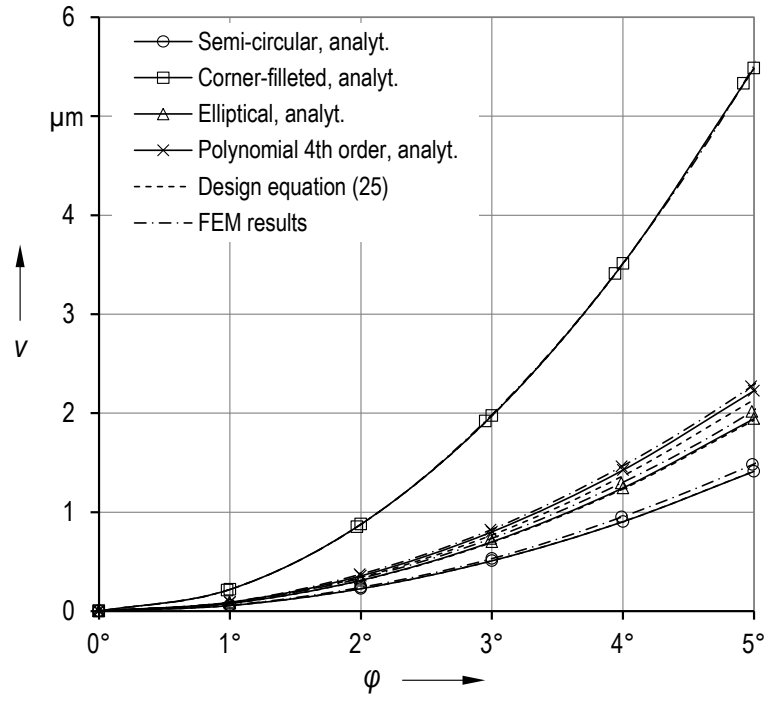

(a)

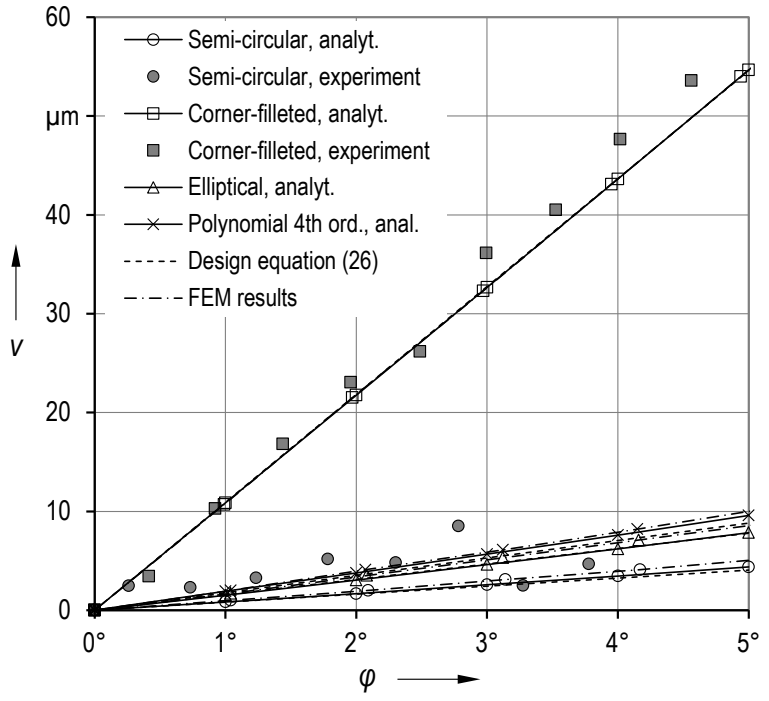

(b)

Figure 23. Comparison of results for the rotational precision of a flexure hinge with various contours $\left(\beta_{l}=1, \beta_{h}=0.03\right)$ : (a) moment load, (b) force load.

In conclusion, the suggested design equations are suitable to predict the deformation and motion behaviour of a flexure hinge in dependence of the hinge contour within the investigated parameter range. The presented design equations are advantageous, because they are concise, and with only two coefficients their structural form is simple, contourindependent and, with the exception of the parameter $H$ for geometric scaling, dimensionless. The principle influence of scaling, which is expressed by the parameter $H$ in the design equations, was also found by means of FEM simulations (Linß and Zentner, 2015). Taken into account a possible in- creasing error, the design equations could be applied for a larger parameter range and rotation angle too.

Regarding the compliant mechanism application, the contour comparison confirms the potential of elliptical and biquadratic polynomial contours to realize a large motion range with high precision. A strong influence of the contour on the flexure hinge performance exists in particular for thin hinges, which are suitable for application due to low strain values and thus larger angular deflections. Furthermore, the use of different flexure hinges in the same mechanism is very promising especially in terms of variable higher order polynomial contours, cf. Eq. (7). In this case, the polynomial order can 
be adjusted easily in dependence of the relative rotation angle in the mechanism by means of using design graphs ( $\operatorname{Lin} \beta$ et al., 2015).

\section{Conclusions}

In this paper, general design equations for the calculation of the rotational stiffness, maximal angular elastic deflection and rotational precision of various notch flexure hinges in dependence of the geometric hinge parameters are suggested and evaluated. The power function based equations are derived for a moment and a transverse force loaded beam by fitting the analytical results which are obtained due to nonlinear modelling with the theory of large deflections of rods. For the accurate model-based investigation of the rotational precision the fixed centre approach is used to define the axis of rotation. Among the variety of existing notch geometries, four flexure hinge contours are selected and investigated: Three usual contours, the semi-circular, corner-filleted and elliptical contour, and the recently introduced bi-quadratic polynomial contour of 4th order, which simultaneously provides a large angular deflection and a high rotational precision. Depending on the contour, the maximum root mean squared error of the calculated results is in the range of less than $2 \%$ to less than $16 \%$ for an appropriate parameter range compared with the analytical solution. Furthermore, the FEM simulations and experimental results correlate well with the predictions based on the design equations. The presented equations are advantageous because with only two coefficients their structural form is simple, concise, contourindependent and dimensionless. Thus, the novel design equations contribute to the accelerated and goal-oriented synthesis of compliant mechanisms with the most commonly used hinge contours or the promising polynomial flexure hinges. More hinge contours, like higher order polynomial contours of a different order, can also be considered by determining the values of their coefficients in further research. 
Appendix A: Circular flexure hinge design equations (adapted to the symbols of this paper)

Paros and Weisbord (1965), simplified expression:

$\frac{M}{\varphi}=\frac{2 E b h^{2.5}}{9 \pi \sqrt{R}}$.

Tseytlin (2002):

$\frac{M}{\varphi}=\frac{E b\left(\frac{h}{2}\right)^{2}}{4\left(1+\sqrt{1+\frac{2 R 0.1986}{h}}\right)}$.

Lobontiu (2003):

$$
\begin{aligned}
& \frac{M}{\varphi}=\frac{E b h^{3}(2 R+h)(4 R+h)^{3}}{24 R} \\
& {\left[h(4 R+h)\left(6 R^{2}+4 R h+h^{2}\right)+6 R(2 r+h)^{2}\right.} \\
& \left.\sqrt{4(4 R+h)} \tan ^{-1}\left(\sqrt{1+\frac{4 R}{h}}\right)\right]^{-1} .
\end{aligned}
$$


Competing interests. The authors declare that they have no conflict of interest.

Acknowledgements. The authors would like to gratefully acknowledge the support of the German Research Foundation (DFG) under Grant no. ZE 714/10-1.

Edited by: G. Hao

Reviewed by: two anonymous referees

\section{References}

Chen, G., Shao, X., and Huang, X.: A new generalized model for elliptical arc flexure hinges, Rev. Sci. Instrum., 79, 95103, doi:10.1063/1.2976756, 2008.

Chen, G., Liu, X., Gao, H., and Jia, J.: A generalized model for conic flexure hinges, Rev. Sci. Instrum., 80, 55106, doi:10.1063/1.3137074, 2009.

Chen, G., Liu, X., and Du, Y.: Elliptical-Arc-Fillet Flexure Hinges: Toward a Generalized Model for Commonly Used Flexure Hinges, J. Mech. Des., 133, 81002, doi:10.1115/1.4004441, 2011.

Chen, G., Wang, J., and Liu, X.: Generalized Equations for Estimating Stress Concentration Factors of Various Notch Flexure Hinges, J. Mech. Des., 136, 031009, doi:10.1115/1.4026265, 2014.

Chen, G.-M., Jia, J.-Y., and Li, Z.-W.: Right-circular corner-filleted flexure hinges, in: IEEE International Conference on Automation Science and Engineering, Edmonton, Canada, 249-253, doi:10.1109/COASE.2005.1506777, 2005.

Christen, G. and Pfefferkorn, H.: Nachgiebige Mechanismen: Aufbau, Gestaltung, Dimensionierung und experimentelle Untersuchung, in: VDI-Berichte Nr. 1423, VDI-Getriebetagung, Kassel, Germany, 309-329, 1998.

De Bona, F. and Munteanu, M. G.: Optimized Flexural Hinges for Compliant Micromechanisms, Analog. Integr. Circ. S., 44, 163174, doi:10.1007/s10470-005-2597-7, 2005.

Desrochers, S.: Optimum design of simplical uniaxial accelerometers, master thesis, McGill University, Montréal, 2008.

Dirksen, F.: Non-intuitive Design of Compliant Mechanisms Possessing Optimized Flexure Hinges, doctoral thesis, Universität der Bundeswehr Hamburg, Hamburg, http://nbn-resolving.de/ urn:nbn:de:gbv:705-opus-30450, 2013.

Dirksen, F. and Lammering, R.: On mechanical properties of planar flexure hinges of compliant mechanisms, Mech. Sci., 2, 109-117, doi:10.5194/ms-2-109-2011, 2011.

Henein, S.: Tutorial on the design of flexure-mechanisms: Flexures: simply subtle, Neuchâtel, FSRM, 2011.

Henein, S., Spanoudakis, P., Droz, S., Myklebust, L. I., and Onillon, E.: Flexure pivot for aerospace mechanisms, in: 10th European Space Mechanisms and Tribology Symposium, San Sebastian, Spain, 2003.

Horie, M., Nozaki, T., Ikegami, K., and Kobayashi, F.: Design System of Superelastic Hinges and Its Application to Micromanipulators, JSME Int. J., 40, 323-328, doi:10.1299/jsmec.40.323, 1997.
Howell, L. L. and Midha, A.: A Method for the Design of Compliant Mechanisms with Small-Length Flexural Pivots, J. Mech. Des., 116, 280-290, doi:10.1115/1.2919359, 1994.

Howell, L. L., Magleby, S. P., and Olsen, B. M.: Handbook of Compliant Mechanisms, Wiley, Chichester, 2013.

Hu, J. F., Li, P., and Cui, X. F.: Optimum Design of a Parabolic Flexure Hinge Based on Compliance ClosedForm Equations, Adv. Mat. Res., 424/425, 299-303, doi:10.4028/www.scientific.net/AMR.424-425.299, 2012.

Kunz, J.: Filmgelenke dehnungsbezogen auslegen, Kunststoffe: Werkstoffe, Verarbeitung, Anwendung, 97, 129-132, 2007.

Li, Q., Pan, C., and Xu, X.: Closed-form compliance equations for power-function-shaped flexure hinge based on unit-load method, Precis. Eng., 37, 135-145, doi:10.1016/j.precisioneng.2012.07.010, 2013.

Lin, R., Zhang, X., Long, X., and Fatikow, S.: Hybrid flexure hinges, Rev. Sci. Instrum., 84, 085004, doi:10.1063/1.4818522, 2013.

Linß, S.: Ein Beitrag zur geometrischen Gestaltung und Optimierung prismatischer Festkörpergelenke in nachgiebigen Koppelmechanismen, doctoral thesis, TU Ilmenau, Ilmenau, http: //nbn-resolving.de/urn:nbn:de:gbv:ilm1-2015000283, 2015.

Linß, S. and Zentner, L.: FEM-Based Analysis of the Influence of Notch Contour and Scale on Flexure Hinge Design Goals, in: The 3rd International Conference Mechanical Engineering in XXI Century, Niš, Serbien, 283-286, 2015.

Linß, S., Erbe, T., and Zentner, L.: On polynomial flexure hinges for increased deflection and an approach for simplified manufacturing, in: 13th World Congress in Mechanism and Machine Science, Guanajuato, Mexico, A11_512, available at: http: //www.dmg-lib.org/dmglib/handler?docum=22411009, (last access: 24 November 2016), 2011 a.

Linß, S., Erbe, T., Theska, R., and Zentner, L.: The influence of asymmetric flexure hinges on the axis of rotation, in: 56th International Scientific Colloquium, Ilmenau, Germany, http:// nbn-resolving.de/urn:nbn:de:gbv:ilm1-2011iwk-006:6, $2011 \mathrm{~b}$.

Linß, S., Milojevic, A., and Zentner, L.: Considering the Design of the Flexure Hinge Contour for the Synthesis of Compliant Linkage Mechanisms, in: 58th Ilmenau Scientific Colloquium, Ilmenau, Germany, http://nbn-resolving.de/urn:nbn:de: gbv:ilm1-2014iwk-033:4, 2014.

Linß, S., Milojevic, A., Pavlovic, N. D., and Zentner, L.: Synthesis of Compliant Mechanisms based on GoalOriented Design Guidelines for Prismatic Flexure Hinges with Polynomial Contours, in: 14th World Congress in Mechanism and Machine Science, Taipei, Taiwan, doi:10.6567/IFToMM.14TH.WC.PS10.008, 2015.

Lobontiu, N.: Compliant Mechanisms: Design of Flexure Hinges, CRC Press, Boca Raton, Fla., 2003.

Lobontiu, N., Cullin, M., Ali, M., and Brock, J. M.: A generalized analytical compliance model for transversely symmetric three-segment flexure hinges, Rev. Sci. Instrum., 82, 105116, doi:10.1063/1.3656075, 2011.

Martin, J. and Robert, M.: Novel Flexible Pivot with Large Angular Range and Small Center Shift to be Integrated into a BioInspired Robotic Hand, J. Intell. Mat. Syst. Str., 22, 1431-1437, doi:10.1177/1045389X11412639, 2011. 
Meng, Q., Li, Y., and Xu, J.: New empirical stiffness equations for corner-filleted flexure hinges, Mech. Sci., 4, 345-356, doi:10.5194/ms-4-345-2013, 2013.

Palmieri, G., Palpacelli, M. C., and Callegari, M.: Study of a Fully Compliant U-Joint Designed for Minirobotics Applications, J. Mech. Des.-T. ASME, 134, 111003-111004, doi:10.1063/1.3137074, 2012.

Paros, J. M. and Weisbord, L.: How to design flexure hinges, Machine design, 25, 151-156, doi:10.1063/1.3137074, 1965.

Pavlović, N. D., Petkovic, D., and Pavlovic, N. T.: Optimal selection of the compliant mechanism synthesis method, in: The International Conference Mechanical Engineering in XXI Century, Niš, Serbien, 247-250, 2010.

Pei, X., Yu, J., Zong, G., and Bi, S.: The modeling of leaf-type isosceles-trapezoidal flexural pivots, in: ASME 2007 International Design Engineering Technical Conferences \& Computers and Information in Engineering Conference, Las Vegas, Nevada, 217-223, doi:10.1115/DETC2007-34981, 2007.

Schotborgh, W. O., Kokkeler, F. G., Tragter, H., and Houten, F. J. A. M. van: Dimensionless design graphs for flexure elements and a comparison between three flexure elements, Precis. Eng., 29, 4147, doi:10.1016/j.precisioneng.2004.04.003, 2005.

Smith, S. T.: Flexures: elements of elastic mechanisms, Gordon \& Breach, Amsterdam, 2000

Smith, S. T., Badami, V. G., Dale, J. S., and Xu, Y.: Elliptical flexure hinges, Rev. Sci. Instrum., 68, 1474-1483, doi:10.1063/1.1147635, 1997.

Tian, Y., Shirinzadeh, B., Zhang, D., and Zhong, Y.: Three flexure hinges for compliant mechanism designs based on dimensionless graph analysis, Precis. Eng., 34, 92-101, doi:10.1016/j.precisioneng.2009.03.004, 2010.

Tres, P. A.: Designing plastic parts for assembly, Hanser/Gardner, Munich, Cincinnati, 1995.

Tseytlin, Y. M.: Notch flexure hinges: An effective theory, Rev. Sci. Instrum., 73, 3363-3368, doi:10.1063/1.1499761, 2002.
Vallance, R. R., Haghighian, B., and Marsh, E. R.: A unified geometric model for designing elastic pivots, Precis. Eng., 32, 278288, doi:10.1016/j.precisioneng.2007.10.001, 2008.

Venanzi, S., Giesen, P., and Parenti-Castelli, V.: A novel technique for position analysis of planar compliant mechanisms, Mech. Mach. Theory, 40, 1224-1239, doi:10.1016/j.mechmachtheory.2005.01.009, 2005.

Wang, R., Zhou, X., and Zhu, Z.: Development of a novel sort of exponent-sine-shaped flexure hinges, Rev. Sci. Instrum., 84, 095008, doi:10.1063/1.4821940, 2013.

Wittwer, J. W. and Howell, L. L.: Mitigating the Effects of Local Flexibility at the Built-In Ends of Cantilever Beams, J. Appl. Mech, 71, 748-751, doi:10.1115/1.1782913, 2004.

Wu, Y. and Zhou, Z.: Design calculations for flexure hinges, Rev. Sci. Instrum., 73, 3101, doi:10.1063/1.1494855, 2002.

Yong, Y. K., Lu, T.-F., and Handley, D. C.: Review of circular flexure hinge design equations and derivation of empirical formulations, Precis. Eng., 32, 63-70, doi:10.1016/j.precisioneng.2007.05.002, 2008.

Zelenika, S., Munteanu, M. G., and De Bona, F.: Optimized flexural hinge shapes for microsystems and highprecision applications, Mech. Mach. Theory, 44, 1826-1839, doi:10.1016/j.mechmachtheory.2009.03.007, 2009.

Zentner, L.: Nachgiebige Mechanismen, De Gruyter Oldenbourg, München, 2014.

Zettl, B., Szyszkowski, W., and Zhang, W. J.: On Systematic Errors of Two-Dimensional Finite Element Modeling of Right Circular Planar Flexure Hinges, J. Mech. Des.-T. ASME, 127, 782-787, doi:10.1115/1.1898341, 2005.

Zhang, Z. and $\mathrm{Hu}, \mathrm{H}$.: Accurate Equivalent Beam Model of a Planar Compliant Mechanism with Elliptical Flexure Hinges, in: International Conference on Measuring Technology and Mechatronics Automation, Zhangjiajie, Hunan, China, 11-14, doi:10.1109/ICMTMA.2009.363, 2009.

Zhu, B. L., Zhang, X. M., and Fatikow, S.: Design of single-axis flexure hinges using continuum topology optimization method, Sci. China Technol. Sci., 57, 560-567, doi:10.1007/s11431-0135446-4, 2014. 